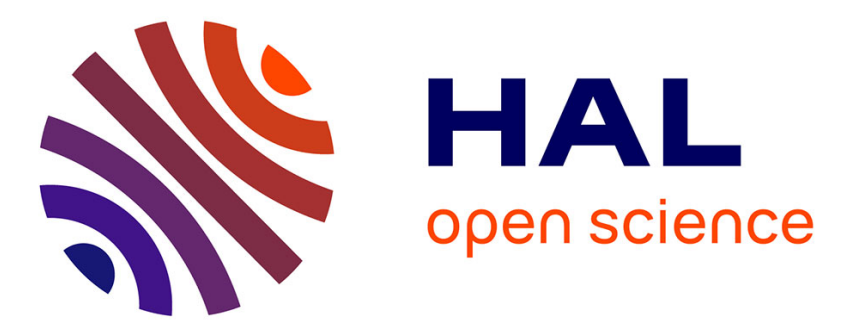

\title{
Entropy formulation of degenerate parabolic equation with zero-flux boundary condition
}

\author{
Boris Andreianov, Mohamed Karimou Gazibo
}

\section{To cite this version:}

Boris Andreianov, Mohamed Karimou Gazibo. Entropy formulation of degenerate parabolic equation with zero-flux boundary condition. Journal of Applied Mathematics and Mechanics / Zeitschrift für Angewandte Mathematik und Mechanik, 2013, 164 (5), pp. 1471-1491. 10.1007/s00033-012-0297-6 . hal-00697593v2

\section{HAL Id: hal-00697593 \\ https://hal.science/hal-00697593v2}

Submitted on 4 Oct 2012

HAL is a multi-disciplinary open access archive for the deposit and dissemination of scientific research documents, whether they are published or not. The documents may come from teaching and research institutions in France or abroad, or from public or private research centers.
L'archive ouverte pluridisciplinaire HAL, est destinée au dépôt et à la diffusion de documents scientifiques de niveau recherche, publiés ou non, émanant des établissements d'enseignement et de recherche français ou étrangers, des laboratoires publics ou privés.

\section{다)(1) $(5$}

Distributed under a Creative Commons Attribution - NonCommerciall 4.0 International 


\title{
Entropy formulation of degenerate parabolic equation with zero-flux boundary condition
}

\author{
Boris Andreianov and Mohamed Karimou Gazibo
}

\begin{abstract}
We consider the general degenerate hyperbolic-parabolic equation:

$$
u_{t}+\operatorname{div} f(u)-\Delta \phi(u)=0 \text { in } Q=(0, T) \times \Omega, \quad T>0, \quad \Omega \subset \mathbb{R}^{N}
$$

with initial condition and the zero flux boundary condition. Here $\phi$ is a continuous non decreasing function. Following [Bürger, Frid and Karlsen, J. Math. Anal. Appl, 2007], we assume that $f$ is compactly supported (this is the case in several applications) and we define an appropriate notion of entropy solution. Using vanishing viscosity approximation, we prove existence of entropy solution for any space dimension $N \geq 1$ under a partial genuine nonlinearity assumption on $f$. Uniqueness is shown for the case $N=1$, using the idea of [Andreianov and Bouhsiss, J. Evol. Equ., 2004], nonlinear semigroup theory and a specific regularity result for one dimension.
\end{abstract}

Mathematics Subject Classification (2010). Primary 35F31; Secondary 00A69.

Keywords. Degenerate hyperbolic-parabolic equation; Zero-flux boundary condition; Vanishing viscosity approximation; Nonlinear semigroup theory; Entropy solution; Integral solution.

\section{Introduction}

Let $\Omega$ be a bounded open set of $\mathbb{R}^{N}$ with a Lipschitz boundary $\partial \Omega$ and $\eta$ the unit normal to $\partial \Omega$ outward to $\Omega$. We consider the zero-flux boundary problem:

$$
(P)\left\{\begin{aligned}
u_{t}+\operatorname{div} f(u)-\Delta \phi(u) & =0 & & \text { in } \quad Q=(0, T) \times \Omega, \\
u(0, x) & =u_{0}(x) & & \text { in } \quad \Omega, \\
(f(u)-\nabla \phi(u)) \cdot \eta & =0 & & \text { on } \quad \Sigma=(0, T) \times \partial \Omega .
\end{aligned}\right.
$$

We assume that the convection flux $f$ is a Lipschitz continuous function. Moreover, we require that

$$
f(0)=0, \quad f\left(u_{\max }\right)=0 \text { for some } u_{\max }>0 .
$$

Accordingly, the initial datum is a mesurable function taking values in the interval $\left[0, u_{\max }\right]$, which will be the invariant domain for the solutions of $(P)$ under assumption (1.1). With a slight abuse of terminology, we will say that $f$ is compactly supported in order to refer to (1.1) along with the choice of $\left[0, u_{\max }\right]$-valued data. Further, the function $\phi$ is continuous non decreasing on $\left[0, u_{\max }\right]$. This assumption means that the problem $(P)$ is of degenerate parabolic-hyperbolic type. For the sake of simplicity, we will treat the case where $\phi($.$) is constant on \left[0, u_{c}\right]$ with $0 \leq u_{c} \leq u_{\max }$ and $\phi($.$) is$ strictly increasing on $\left[u_{c}, u_{\max }\right]$. The case of a general $\phi$ can be treated without additional difficulty (see Carrillo [13]).

The framework (E) includes hyperbolic conservation law as a particular case and it is well known that in general, global classical solutions may not exist; and that weak solution in the sense of distributions may not be unique. The standard way to fix this problem is to work with the socalled entropy solution (see Kruzhkov [15] for the case of conservation laws, and Carrillo [13] for the adaptation of this notion to the case of degenerate elliptic-parabolic-hyperbolic equation). There 
exist many papers in the literature dealing with Dirichlet boundary condition for (E). The main reference is the fundamental paper of Carrillo for homogenous Dirichlet boundary condition [13] which establishes the uniqueness technique. In [21], Rouvre and Gagneux prove also existence and uniqueness for homogenous Dirichlet condition under strong regularity requirement on the data. The general Dirichlet boundary condition received much attention, see Mascia and al [17], Michel and Vovelle [19], Vallet [22]. However, the Dirichlet boundary condition may not always provide the most natural setting for this kind of problem on bounded domains. Equation (E) occurs in several applications, for example it comes from the theory of porous media flow, phenomenological theory of sedimentation-consolidation processes, road traffic. In practice, it is often supplemented with the zero-flux (homogeneous Neumann boundary condition), at least on a part of the boundary (see [11]).

Let us describe in more detail one application. Problem $(P)$ is of interest in describing pressure filtration of flocculated suspensions. The domain $\Omega$ is a filter medium, which lets only the liquid pass, by a piston which moves downwards due to an applied pressure. The material behavior of the suspension is described by two model functions, the flux density function or hindered settling factor $f$ and the effective solid stress function $\phi$, both functions only of the local solids concentration $u$. Here $f$ is a nonpositive Lipschitz continuous function with compact support in $\left[0, u_{\max }\right]$, where $u_{\max } \leq 1$ is the maximum concentration and the function $\phi$ satisfies $\phi(u)=0$ for $u \leq u_{c}$, with $0 \leq u_{c} \leq u_{\max }$, where $u_{c}$ is a critical concentration value, and $\phi^{\prime}(u)>0$ for $u>u_{c}$. Notice that these assumptions are exactly those that we have taken in this paper. According to the phenomenological sedimentation-consolidation theory [11], the evolution of the concentration distribution is subject to Neumann boundary condition at least on a part of the boundary, and this is our motivation.

In [10] Bürger and al. consider the problem $(P)$ with $\phi(u) \equiv 0$. They introduce a notion of entropy solution based on the existence of strong trace $u^{\tau}$ on $\partial \Omega$ under some assumption on the boundary (see [23]) and the flux $f$ which satisfies (1.1). They prove existence and uniqueness of entropy solution. The purpose of our paper is to extend the result of Bürger and al. ([10]) to degenerate parabolic-hyperbolic equation. The extension is not trivial, and as a matter of fact, we are unable to prove uniqueness in dimension $N \geq 2$ although we believe that the notion of entropy solution we introduce in this paper is relevant for any dimension. Let us explain the difficulties and the techniques we use to overcome them. Since the total flux in (E) contains the diffusion flux term $\nabla \phi(u)$ which is only $L^{2}$, we cannot ensure the existence of a strong trace for this term. Therefore, we suppose that this boundary condition is satisfied in the weak sense only. We propose a new entropy formulation that incorporates a boundary term which does not contain any trace of $u$. Its main advantage over the definition of [10] is that the stability under the $L^{1}(Q)$ convergence of solutions is evident. Notice that we do not need existence of traces of entropy solutions $u$ of (E), even if it could be ensured.

To prove existence of entropy solution, we use a classical vanishing viscosity approximation and get the a priori estimates useful for passing to the limit in the approximate problem. The main point for passing to the limit is based on a rather involved local compacity argument of Panov [20]. We manage to apply this result in our case and prove that the limit of entropy solutions of approximate problem is an entropy solution of $(P)$.

Now, let us focus on the question of uniqueness of entropy solution for $(P)$. For this aim, we prove a version of an important proposition due to Carrillo [13]. This proposition identifies the entropy dissipation term which is a key ingredient of the uniqueness technique. Then, it is easy to prove uniqueness of solutions such that the boundary condition is satisfied in the sense of strong boundary trace of the normal component of the flux $(f(u)-\nabla \phi(u))$. Unfortunately, we are able to establish this additional solution regularity only for the stationary problem $(S)$ associated to $(P)$ (see section 4 ) and only in the case of one space dimension. Therefore, we adapt the hint from the paper [2] (see also [3]) and compare a general solution to $(P)$ with a regular solution to $(S)$. We conclude by a standard application of the notion of integral solution coming from the nonlinear semigroup theory [6]. Eventually, we prove the uniqueness result in space dimension one.

Let us stress that the problem of uniqueness is still open in multiple space dimensions. Uniqueness of regular solutions to $(P)$ is trivially true, and the abscence of regularity near the boundary makes 
the problem technically very delicate. The definition of strong traces of the solution with respect to the lateral boundary of the domain $\Omega$ is possible if for example the diffusion term $\phi(u)$ is such that $f(u)-\nabla \phi(u)$ is continuous up to the boundary $\partial \Omega$. If there existed "sufficiently many" solutions (in the sense of [2], [3]) having this regularity, uniqueness would follow, by comparaison of a general solution with an ad hoc sequence of regular solutions. We leave the investigation of this regularity question to a future work. Another open question is how to define entropy solutions in the case where assumption (1.1) does not hold. Indeed, as in [10], assumption (1.1) ensures that the zero-flux boundary condition is satisfied literally. When this assumption is dropped, we expect that the boundary condition should be relaxed, as in the case of Dirichlet boundary condition (see [5]). One example for the zero-flux hyperbolic problem is given in [4].

The rest of this paper is organized as follows. In section 2, we give some assumptions and preliminaries and state our definition of entropy solution. Section 3 is devoted to existence of approximate solutions and passage to the limit to prove existence of an entropy solution of $(P)$. Finally, in section 4 we study the abstract evolution equation associated with $(P)$ and prove uniqueness of entropy solution in one space dimension.

\section{Entropy Solution}

\subsection{Assumptions and preliminaries}

We introduce the sign function and its approximations:

$$
\begin{aligned}
& \operatorname{sign}(r)=\left\{\begin{array}{rl}
1 & \text { if } r>0, \\
0 & \text { if } r=0, \\
-1 & \text { if } r<0,
\end{array} \operatorname{sign}^{+}(r)=\left\{\begin{array}{ll}
1 & \text { if } r>0, \\
0 & \text { if } r \leq 0,
\end{array} \quad \operatorname{sign}^{-}(r)=\left\{\begin{array}{rr}
0 & \text { if } r>0, \\
-1 & \text { if } r \leq 0,
\end{array}\right.\right.\right. \\
& \operatorname{sign}_{\sigma}(r)=\left\{\begin{aligned}
1 & \text { if } r>\sigma, \\
\frac{r}{\sigma} & \text { if }|r| \leq \sigma, \\
-1 & \text { if } r<-\sigma,
\end{aligned}\right.
\end{aligned}
$$

We also introduce the cut-off function:

$T_{a, b}(r)=\left\{\begin{array}{l}a \text { if } r<a, \\ r \text { if } a \leq r \leq b, \\ b \text { if } r>b\end{array}\right.$

To apply a strong precompactness result needed for the proof of the existence of entropy solution, we assume that the couple $(f(),. \phi()$.$) is non-degenerate in the sense of the following definition.$

Definition 2.1. (Panov [20]). Let $\phi$ be zero on $\left[0, u_{c}\right]$, strictly increasing on $\left[u_{c}, u_{\max }\right]$ and a vector $f=\left(f_{1}, \ldots, f_{N}\right)$. A couple $(f(),. \phi()$.$) is said to be non-degenerate if, for all \xi \in \mathbb{R}^{N} \backslash\{0\}$, the functions $\lambda \longmapsto \sum_{i=1}^{N} \xi_{i} f_{i}(\lambda)$ are not "affine" on the non-degenerate sub intervals of $\left[0, u_{c}\right]$.

\subsection{Definition of Entropy Solution}

In this section, we give our entropy formulation for the problem $P$.

Definition 2.2. Let $u_{0}$ be a measurable $\left[0, u_{\max }\right]$-valued function. A measurable function $u$ taking values on $\left[0, u_{\max }\right]$ is called weak solution of problem $(P)$ if $: \phi(u) \in L^{2}\left(0, T ; H^{1}(\Omega)\right)$ and for all $\xi \in L^{2}\left(0, T ; H^{1}(\Omega)\right)$ such that $\xi_{t} \in L^{1}(Q)$ and $\xi(T,)=$.0 , one has

$$
\int_{0}^{T} \int_{\Omega}\left\{u \xi_{t}+(f(u)-\nabla \phi(u)) \cdot \nabla \xi\right\} d x d t+\int_{\Omega} u_{0} \xi(0, x) d x=0 .
$$

Definition 2.3. A measurable function $u$ taking values on $\left[0, u_{\max }\right]$ is called an entropy solution of the initial-boundary value problem $(P)$ if $\phi(u) \in L^{2}\left(0, T ; H^{1}(\Omega)\right)$ and $\forall k \in\left[0, u_{\max }\right], \forall \xi \in \mathcal{C}^{\infty}\left([0, T) \times \mathbb{R}^{N}\right)$, 
with $\xi \geq 0$, the following inequality hold

$$
\begin{aligned}
& \int_{0}^{T} \int_{\Omega}\left\{|u-k| \xi_{t}+\operatorname{sign}(u-k)[f(u)-f(k)-\nabla \phi(u)] . \nabla \xi\right\} d x d t \\
& +\int_{0}^{T} \int_{\partial \Omega}|f(k) \cdot \eta(x)| \xi(t, x) d \mathcal{H}^{N-1} d t+\int_{\Omega}\left|u_{0}-k\right| \xi(0, x) d x \geq 0 .
\end{aligned}
$$

If we remplace (2.2) by one of the following inequalities

$$
\begin{aligned}
& \int_{0}^{T} \int_{\Omega}\left\{(u-k)^{+} \xi_{t}+\operatorname{sign}^{+}(u-k)[f(u)-f(k)-\nabla \phi(u)] \cdot \nabla \xi\right\} d x d t \\
& +\int_{0}^{T} \int_{\partial \Omega}(f(k) \cdot \eta(x))^{+} \xi(t, x) d \mathcal{H}^{N-1} d t+\int_{\Omega}\left(u_{0}-k\right)^{+} \xi(0, x) d x \geq 0, \\
& \int_{0}^{T} \int_{\Omega}\left\{(u-k)^{-} \xi_{t}+\operatorname{sign}^{-}(u-k)[f(u)-f(k)-\nabla \phi(u)] \cdot \nabla \xi\right\} d x d t \\
& +\int_{0}^{T} \int_{\partial \Omega}(f(k) \cdot \eta(x))^{-} \xi(t, x) d \mathcal{H}^{N-1} d t+\int_{\Omega}\left(u_{0}-k\right)^{-} \xi(0, x) d x \geq 0,
\end{aligned}
$$

we obtain notions of entropy sub-solution and entropy super-solution respectively. Obviously, a function $u$ is an entropy solution if and only if $u$ is entropy sub-solution and entropy super-solution simultaneously.

Remark 2.4. 1. For the case $\phi=0$, solution of [10] is solution in our sense. The converse assertion is also true at least for $N=1$, this is the consequence of the uniqueness of a solution in the sense of Definition 2.3.

2. The entropy solution in the sense of Definition 2.3 is in particular a weak solution in the sense of Definition 2.2. Indeed, take in (2.2) $k=0$ and $k=u_{\max }$ and use (1.1); we find (2.1).

3. Let us stress that, in particular, the zero flux boundary condition $(f(u)-\nabla \phi(u)) \cdot \eta=0$ is verified literally in the weak sense. This contrasts with the properties of the Dirichlet problem (see [5]); we expect that the boundary condition should be relaxed if assumption (1.1) is dropped (see [4]).

\section{Existence of Entropy Solutions}

The main result of this section is the following theorem:

Theorem 3.1. Assume that (1.1) holds and $(f, \phi)$ is non-degenerate in the sense of Definition 2.3. Then there exists an entropy solution $u$ for the problem $(P)$.

\subsection{Viscosity Regularized Problem}

To show the existence of entropy solution, we approximate $\phi(u)$ by $\phi_{\epsilon}\left(u^{\epsilon}\right)=\phi\left(u^{\epsilon}\right)+\epsilon I d\left(u^{\epsilon}\right)$ for each $\epsilon>0$. We obtain the following regularized problem $\left(P_{\epsilon}\right)$ :

$$
\left(P_{\epsilon}\right)\left\{\begin{aligned}
u_{t}^{\epsilon}+\operatorname{div} f\left(u^{\epsilon}\right)-\Delta \phi_{\epsilon}\left(u^{\epsilon}\right) & =0 & & \text { in } \quad Q=(0, T) \times \Omega, \\
u^{\epsilon}(0, x) & =u_{0}^{\epsilon}(x) & & \text { in } \quad \Omega, \\
\left(f\left(u^{\epsilon}\right)-\nabla \phi_{\epsilon}\left(u^{\epsilon}\right)\right) \cdot \eta & =0 & & \text { on } \quad \Sigma=(0, T) \times \partial \Omega,
\end{aligned}\right.
$$

where $\left(u_{0}^{\epsilon}\right)_{\epsilon}$ is a sequence of smooth functions that converges to $u_{0}$ a.e and respects the minimum/maximum values of $u_{0}$.

Definition 3.2. A function $u^{\epsilon} \in L^{2}\left(0, T ; H^{1}(\Omega)\right)$ is called weak solution of the initial-boundary value problem $\left(P_{\epsilon}\right)$ if for all $\xi \in L^{2}\left(0, T ; H^{1}(\Omega)\right)$ such that $\xi_{t} \in L^{1}(Q)$ and $\xi(T,)=$.0 , one has

$$
\int_{0}^{T} \int_{\Omega}\left\{u^{\epsilon} \xi_{t}+\left(f\left(u^{\epsilon}\right)-\nabla \phi_{\epsilon}\left(u^{\epsilon}\right)\right) \cdot \nabla \xi\right\} d x d t+\int_{\Omega} u_{0}^{\epsilon} \xi(0, x) d x=0 .
$$


Definition 3.3. A measurable function $u^{\epsilon} \in L^{2}\left(0, T ; H^{1}(\Omega)\right)$ taking values in $\left[0, u_{\max }\right]$ is called an entropy solution of $\left(P_{\epsilon}\right)$ if $\forall k \in\left[0, u_{\max }\right], \forall \xi \in \mathcal{C}^{\infty}\left([0, T) \times \mathbb{R}^{N}\right), \xi \geq 0$, the following inequality hold

$$
\begin{aligned}
& \int_{0}^{T} \int_{\Omega}\left\{\left|u^{\epsilon}-k\right| \xi_{t}+\operatorname{sign}\left(u^{\epsilon}-k\right)\left[f\left(u^{\epsilon}\right)-f(k)-\nabla \phi_{\epsilon}\left(u^{\epsilon}\right)\right] . \nabla \xi\right\} d x d t \\
& +\int_{0}^{T} \int_{\partial \Omega}|f(k) \cdot \eta(x)| \xi(t, x) d \mathcal{H}^{N-1} d t+\int_{\Omega}\left|u_{0}^{\epsilon}-k\right| \xi(0, x) d x \geq 0 .
\end{aligned}
$$

Theorem 3.4. Assume that $u_{0} \in\left[0, u_{\max }\right]$ and (1.1) holds. Then the problem $\left(P_{\epsilon}\right)$ admits a weak solution $u^{\epsilon}$ which is also an entropy solution. In particular, we have $0 \leq u^{\epsilon} \leq u_{\max }$. In addition, there exists $C$ independent on $\epsilon$ such that

$$
\begin{gathered}
\left\|\sqrt{\epsilon} \nabla u^{\epsilon}\right\|_{L^{2}(Q)} \leq C ; \\
\left\|\phi_{\epsilon}\left(u_{\epsilon}\right)\right\|_{L^{2}\left(0, T ; H^{1}(\Omega)\right)} \leq C .
\end{gathered}
$$

\subsection{Strong pre-compactness result and passage to the limit in $\epsilon$}

Theorem 3.5. (Panov [20]). Assume that $(f, \phi)$ is non degenerate in the sense of Definition 2.1. Suppose $u^{\epsilon}, \epsilon>0$, is a sequence such that

$$
\begin{aligned}
& \exists d>1, \forall a, b \in \mathbb{R} \text { with } a<b \\
& T_{a, b}\left(u^{\epsilon}\right)_{t}+\operatorname{div}\left(f\left(T_{a, b}\left(u^{\epsilon}\right)\right)-\nabla \phi\left(T_{a, b}\left(u^{\epsilon}\right)\right)\right) \text { is pre-compact in } W_{\mathrm{Loc}}^{-1, d}(Q) .
\end{aligned}
$$

Moreover, suppose $u^{\epsilon}, f\left(u^{\epsilon}\right), \phi_{\epsilon}\left(u^{\epsilon}\right)$ are equi-integrable locally on $Q$. Then, there exists a subsequence $\left(T_{a, b}\left(u^{\epsilon}\right)\right)_{\epsilon}$ that converges in $L_{\text {Loc }}^{1}(Q)$.

To prove Theorem 3.5, we need the following result.

Lemma 3.6. Suppose $(f, \phi)$ is non degenerate and let $u^{\epsilon}=u^{\epsilon}(t, x)$ be an entropy solution of $\left(P_{\epsilon}\right)$. Then for all $a, b \in \mathbb{R}$ such that $0 \leq a<b \leq u_{\max }$,

$$
T_{a, b}\left(u^{\epsilon}\right)_{t}+\operatorname{div}\left(f\left(T_{a, b}\left(u^{\epsilon}\right)\right)-\nabla \phi_{\epsilon}\left(T_{a, b}\left(u^{\epsilon}\right)\right)\right)=\kappa_{a, b}^{\epsilon} \text { in } \mathcal{D}^{\prime}(Q)
$$

with $\kappa_{a, b}^{\epsilon} \in M_{b}(Q)$. Here $M_{b}(Q)$ represents the set of all Radon measures on $Q$. Moreover, for each compact set $K \subset Q$, we have $\operatorname{Vark}_{a, b}^{\epsilon}(K) \leq C(K, a, b)$, uniformly in $\epsilon \in(0,1)$.

Proof. By the well known representation property for non-negative distributions, we derive from (3.2) that for each $k \in\left[0, u_{\max }\right]$

$$
\left|u^{\epsilon}-k\right|_{t}+\operatorname{div}\left[\operatorname{sign}\left(u^{\epsilon}-k\right)\left(f\left(u^{\epsilon}\right)-f(k)\right)-\nabla\left|\phi_{\epsilon}\left(u^{\epsilon}\right)-\phi_{\epsilon}(k)\right|\right]=-\kappa_{k}^{\epsilon} \text { in } \mathcal{D}^{\prime}(Q)
$$

where $\kappa_{k}^{\epsilon} \in M_{b}(Q), \kappa_{k}^{\epsilon} \geq 0$. Further, for a compact set $K \subset Q$ we choose a non-negative function $\xi=\xi_{K}(t, x) \in \mathcal{C}_{0}^{\infty}(Q)$, which equals 1 on $K$. Then, we have the estimate

$$
\begin{aligned}
& \kappa_{k}^{\epsilon}(K) \leq \int_{0}^{T} \int_{\Omega} \xi(t, x) d \kappa_{k}^{\epsilon}(t, x) \\
& =\int_{Q}\left\{\left|u^{\epsilon}-k\right| \xi_{t}+\left[\operatorname{sign}\left(u^{\epsilon}-k\right)\left(f\left(u^{\epsilon}\right)-f(k)\right)-\nabla\left|\phi_{\epsilon}\left(u^{\epsilon}\right)-\phi_{\epsilon}(k)\right|\right] \cdot \nabla \xi\right\} \\
& \leq \int_{Q}\left(J_{1}^{\epsilon}+J_{2}\right) \max \left(\left|\xi_{t}\right|,|\nabla \xi|,|\Delta \xi|\right) d x d t=: A\left(K, k, J_{1}^{\epsilon}\right),
\end{aligned}
$$

where $J_{1}^{\epsilon}(t, x)=\left|u^{\epsilon}\right|+\left|f\left(u^{\epsilon}\right)\right|+\left|\phi_{\epsilon}\left(u^{\epsilon}\right)\right|$ and $J_{2}=|k|+|f(k)|+\left|\phi_{\epsilon}(k)\right|$ are bounded in $L^{1}$ due to the fact that $u^{\epsilon}, f\left(u^{\epsilon}\right), \phi_{\epsilon}\left(u^{\epsilon}\right)$ are bounded in $L^{\infty}$, uniformly on $\epsilon$ (see Theorem 3.4). Therefore $\kappa_{k}^{\epsilon}(K)$ 
can be upper bounded by some quantity $A(K, k)$. Further, notice that for each $a, b \in \mathbb{R}$ and for any function $g$

$$
\begin{aligned}
g\left(T_{a, b}(r)\right) & =\frac{1}{2}(\operatorname{sign}(r-a)(g(r)-g(a))-\operatorname{sign}(r-b)(g(r)-g(b))) \\
& +\frac{1}{2}(g(a)+g(b)) .
\end{aligned}
$$

From (3.5), we have with $g=I d, g=f$ and $g=\phi_{\epsilon}$

$$
T_{a, b}\left(u^{\epsilon}\right)_{t}+\operatorname{div}\left(f\left(T_{a, b}\left(u^{\epsilon}\right)\right)-\nabla \phi_{\epsilon}\left(T_{a, b}\left(u^{\epsilon}\right)\right)\right)=\kappa_{a, b}^{\epsilon} \text { in } \mathcal{D}^{\prime}(Q),
$$

with $\kappa_{a, b}^{\epsilon}=\frac{1}{2}\left(\kappa_{b}-\kappa_{a}\right)$. Moreover, we have

$$
\operatorname{Var} \kappa_{a, b}^{\epsilon}(K) \leq \frac{1}{2}(A(K, a)+A(K, b))=: C(K, a, b) .
$$

This concludes the proof.

Notice that for all $a, b \in \mathbb{R}, a<b$, we have $T_{a, b}\left(T_{0, u_{\max }}\right)=T_{\tilde{a}, \tilde{b}}$ with $\tilde{a}=\max (a, 0)$ and $\tilde{b}=\min \left(b, u_{\max }\right)$. In order to justify the passage to the limit, we need the following easy lemma:

Lemma 3.7. Suppose that for all compact set $K \subset Q$, the sequence $\Psi_{n}$ is bounded in $L^{\infty}(K)$, and converges a.e. to $\Psi$. Assume that the sequence $\left(\Phi_{n}\right)$ converges weakly in $L^{2}(K)$ to $\Phi$. Then $\Psi_{n} \Phi_{n}$ converges to $\Psi \Phi$ weakly in $L^{2}(K)$.

Now, we are able to prove Theorem 3.1.

Proof of Theorem 3.1. Take some countable set of values $\epsilon \rightarrow 0$. We derive from Lemma 3.6 and the above remark, that for all $a, b \in \mathbb{R}, a<b$,

$$
T_{a, b}\left(u^{\epsilon}\right)_{t}+\operatorname{div}\left(f\left(T_{a, b}\left(u^{\epsilon}\right)\right)-\nabla \phi\left(T_{a, b}\left(u^{\epsilon}\right)\right)\right)=\kappa_{a, b}^{\epsilon}+\epsilon \Delta T_{a, b}\left(u^{\epsilon}\right) \text { in } \mathcal{D}^{\prime}(Q) ;
$$

here $\left(\kappa_{a, b}^{\epsilon}\right)_{\epsilon}$ is a bounded sequence in $M_{b}(K)$ for each compact set $K \subset Q$. Moreover, due to (3.3), we have that $\epsilon \Delta T_{a, b}\left(u^{\epsilon}\right)$ tends to zero in $H^{-1}(Q)$. By Sobolev embedding we have $H^{-1}(Q)=W^{-1,2}(Q) \subset$ $W^{-1, d}(Q), d \leq 2$, therefore $\epsilon \Delta T_{a, b}\left(u^{\epsilon}\right)$ tends to zero in $W^{-1, d}(Q)$. Since $M_{b}(K)$ is compactly embedded in $W^{-1, d}(K)$ for each $d \in\left[1, \frac{N+1}{N}\right)$, we see that $T_{a, b}\left(u^{\epsilon}\right)_{t}+\operatorname{div}\left(f\left(T_{a, b}\left(u^{\epsilon}\right)\right)-\nabla \phi\left(T_{a, b}\left(u^{\epsilon}\right)\right)\right)$ is precompact in $W^{-1, d}(K)$. Since $(f, \phi)$ is assumed to be non-degenerate, then applying Theorem 3.5 we find $T_{0, u_{\max }}\left(u^{\epsilon}\right) \longrightarrow T_{0, u_{\max }}(u)$ as $\epsilon \rightarrow 0$ in $L^{1}(K)$, for a subsequence. Covering $Q$ by a countable of compact subsets $K$ and using the Cantor diagonal extraction argument, we get $u^{\epsilon} \rightarrow u$ in $L_{\text {Loc }}^{1}(Q)$ ( and actually in $L^{1}(Q)$, because $u^{\epsilon}$ take their values in $\left.\left[0, u_{\max }\right]\right)$. Extracting a further subsequence if necessary, we can assume that $u^{\epsilon} \longrightarrow u$ a.e. in $Q$ as $\epsilon \rightarrow 0$.

It remains to derive the entropy formulation (2.2) for $u$. Passing to the limit in inequality (3.2), we claim that the limit function $u=u(t, x)$ satisfies the inequality (2.2) for all $k \in\left[0, u_{\max }\right]$ such that the level set $u^{-1}(k)$ has zero measure. Indeed, by the continuity of $f$, we have $\operatorname{sign}\left(u^{\epsilon}-k\right)\left(f\left(u^{\epsilon}\right)-f(k)\right) \longrightarrow$ $\operatorname{sign}(u-k)(f(u)-f(k))$ as $\epsilon \rightarrow 0$ a.e. in $Q$. Further, since $\phi_{\epsilon}\left(u_{\epsilon}\right)$ is bounded in $L^{2}\left(0, T ; H^{1}(\Omega)\right)$ and converges a.e to the limit that is readily identified with $\phi(u)$, we deduce by Lemma 3.7 that $\operatorname{sign}\left(u^{\epsilon}-k\right) \nabla \phi_{\epsilon}\left(u^{\epsilon}\right) \rightarrow \operatorname{sign}(u-k) \nabla \phi(u)$ in $L^{2}(Q)$ for $k$ such that $u^{-1}(k)$ has zero measure. Notice that the set of such values of $k$ is dense in $\left[0, u_{\max }\right]$. It is easy to see that the left-hand side of $(2.2)$ is continuous with respect to $k$, because $\nabla \phi(u)=0$ a.e. on the set $[u=k]$ (see Lemma 4.4 below). Therefore, by density we inherit $(2.2)$ for all $k \in\left[0, u_{\max }\right]$. We conclude that $u(t, x)$ is an entropy solution of $(P)$. 


\section{Uniqueness result for entropy solutions in one space dimension}

The main result of this section is the following theorem:

Theorem 4.1. Suppose that $\Omega=(a, b)$ is a bounded interval of $\mathbb{R}$, then $(P)$ admits a unique entropy solution.

Let us first recall an essential property of entropy solutions, based on the idea of J. Carrillo [13].

Proposition 4.2. Let $\xi \in \mathcal{C}^{\infty}\left(\left[0, T\left[\times \mathbb{R}^{N}\right), \xi \geq 0\right.\right.$. Then for all $k \in\left[u_{c}, u_{\max }\right]$, for all $D \in \mathbb{R}^{N}$ and for all entropy solution $u$ of $(P)$, we have

$$
\begin{aligned}
& \int_{0}^{T} \int_{\Omega}\left\{|u-k| \xi_{t}+\operatorname{sign}(u-k)[f(u)-f(k)-\nabla \phi(u)+D] \cdot \nabla \xi\right\} d x d t \\
& +\int_{\Omega}\left|u_{0}-k\right| \xi(0, x) d x+\int_{0}^{T} \int_{\partial \Omega}|(f(k)-D) \cdot \eta(x)| \xi d \mathcal{H}^{N-1} d t \\
& \geq \lim _{\sigma \rightarrow 0} \frac{1}{\sigma} \iint_{Q \cap\{-\sigma<\phi(u)-\phi(k)<\sigma\}} \nabla \phi(u) \cdot(\nabla \phi(u)-D) \xi .
\end{aligned}
$$

Remark 4.3. Notice that this proposition makes explicit the information on the dissipation. Let us stress that in (2.2), $D=0$ and $k \in\left[0, u_{\max }\right]$ while in (4.1) $D \in \mathbb{R}^{N}$ but $k \in\left[u_{c}, u_{\max }\right]$.

Proposition 4.2 is a key ingredient of the uniqueness technique. To prove this proposition, we need the following remarks. Firstly, for all $u \in\left[0, u_{\max }\right]$ and for all $k \in\left[u_{c}, u_{\max }\right]$, one has $\operatorname{sign}(u-k)=$ $\operatorname{sign}(\phi(u)-\phi(k))$. Secondly, recall

Lemma 4.4. For all entropy solution $u$ of $(P)$, one has:

$\nabla \phi(u)=0$ a.e. on the set $\left\{(t, x) \in Q\right.$ such that $\left.u(t, x) \in\left[0, u_{c}\right]\right\}$.

Proof. This result comes from Marcus and Mizel lemma (cf. [18]) which states that for $p \in(1, \infty)$ and $F$ in $W^{1, p}, \nabla F=0$ a.e on $F^{-1}(\mathcal{N})$, where $\mathcal{N}$ is a set of zero measure on $\mathbb{R}$. Applying this for a.e. $t \in(0, T)$, for $u \in\left[0, u_{c}\right]$, we have $\nabla \phi(u)=0$ on $[\phi(u)]^{-1}\{0\}$ with $\phi(u) \in H^{1}(\Omega)$. Let $E_{N+1}=\left\{(t, x)\right.$ such that $\left.u(t, x) \in\left[0, u_{c}\right]\right\}$ and $E_{N}(t)=\left\{x\right.$ such that $\left.u(t, x) \in\left[0, u_{c}\right]\right\}$. Then, by Fubini theorem, $\left|E_{N+1}\right|=\int_{0}^{T}\left|E_{N}(t)\right| d t=0$.

Proof of Theorem 4.2. Since $u$ is a weak solution of $(P)$, then for all $k \in\left[u_{c}, u_{\max }\right]$ and all $D \in \mathbb{R}^{N}$, $u$ is a weak solution of the following problem:

$$
\left(P_{k}\right)\left\{\begin{aligned}
(u-k)_{t}+\operatorname{div}[(f(u)-\nabla \phi(u))-(f(k)-D)] & =0 & & \text { in } Q, \\
u(0, x)-k & =u_{0}(x)-k & & \text { on } \Omega, \\
((f(u)-\nabla \phi(u))-(f(k)-D)) \cdot \eta & =-(f(k)-D) . \eta & & \text { on } \Sigma .
\end{aligned}\right.
$$

Take the test function $\operatorname{sign}_{\sigma}(\phi(u)-\phi(k)) \xi$ in the weak formulation of this problem with $\xi \in \mathcal{C}^{\infty}([0, T) \times$ $\left.\mathbb{R}^{N}\right)$. Using the formalism of [1], we have

$$
\begin{aligned}
& \int_{0}^{T}\left\langle(u-k)_{t}, \operatorname{sign}_{\sigma}(\phi(u)-\phi(k)) \xi\right\rangle_{H^{1}(\Omega)^{*}, H^{1}(\Omega)} d t \\
& -\int_{0}^{T} \int_{\Omega} \operatorname{sign}_{\sigma}(\phi(u)-\phi(k))[(f(u)-\nabla \phi(u))-(f(k)-D)] \cdot \nabla \xi \\
& -\int_{0}^{T} \int_{\Omega} \xi[(f(u)-\nabla \phi(u))-(f(k)-D)] . \nabla \operatorname{sign}_{\sigma}(\phi(u)-\phi(k)) \\
& -\int_{0}^{T} \int_{\partial \Omega} \operatorname{sign}_{\sigma}(\phi(u)-\phi(k))(f(k)-D) \cdot \eta \xi=0 .
\end{aligned}
$$


By the chain rule (see [1], [14]) the first integral of (4.2) gives:

$$
-\iint_{Q} \int_{k}^{u} \operatorname{sign}_{\sigma}(\phi(r)-\phi(k)) d r \xi_{t}-\int_{\Omega} \int_{k}^{u_{0}} \operatorname{sign}_{\sigma}(\phi(r)-\phi(k)) d r \xi(0, x) .
$$

Using the fact that $k \in\left[u_{c}, u_{\max }\right]$ and passing to the limit as $\sigma$ goes to 0 , we obtain:

$$
\begin{aligned}
& \int_{0}^{T} \int_{\Omega}\left\{\int_{k}^{u} \operatorname{sign}_{\sigma}(\phi(r)-\phi(k)) d r\right\} \xi_{t} d x d t \longrightarrow \int_{0}^{T} \int_{\Omega}|u-k| \xi_{t} d x d t, \\
& \int_{\Omega}\left\{\int_{k}^{u_{0}} \operatorname{sign}_{\sigma}(\phi(r)-\phi(k)) d r\right\} \xi(0, x) d x \longrightarrow \int_{\Omega}\left|u_{0}-k\right| \xi(0, x) d x .
\end{aligned}
$$

After passing to the limit as $\sigma$ goes to 0 in the second integral of (4.2), and using the fact that $k \in\left[u_{c}, u_{\text {max }}\right]$, we obtain the expression

$$
\int_{0}^{T} \int_{\Omega} \operatorname{sign}(u-k)[(f(u)-\nabla \phi(u))-(f(k)-D)] \cdot \nabla \xi .
$$

The third integral of (4.2) can be written as

$$
\iint_{Q} \xi(f(u)-f(k)) . \nabla \operatorname{sign}_{\sigma}(u-k)-\iint_{Q} \xi(\nabla \phi(u)-D) . \nabla \operatorname{sign}_{\sigma}(\phi(u)-\phi(k)) .
$$

By passing to the limit, the integral in the first term goes to 0 , and the second term becomes

$$
\begin{aligned}
& \lim _{\sigma \rightarrow 0} \int_{0}^{T} \int_{\Omega} \xi(\nabla \phi(u)-D) \cdot \nabla \operatorname{sign}_{\sigma}(\phi(u)-\phi(k)) \\
& =\lim _{\sigma \rightarrow 0} \int_{0}^{T} \int_{\Omega} \xi(\nabla \phi(u)-D) \operatorname{sign}_{\sigma}^{\prime}(\phi(u)-\phi(k)) \cdot \nabla \phi(u) \\
& =\lim _{\sigma \rightarrow 0} \frac{1}{\sigma} \int_{Q \cap\{-\sigma<\phi(u)-\phi(k)<\sigma\}} \nabla \phi(u) \cdot(\nabla \phi(u)-D) \xi .
\end{aligned}
$$

The limit of last integral of (4.2) can be upper bounded as follow:

$$
\lim _{\sigma \rightarrow 0} \int_{0}^{T} \int_{\partial \Omega} \operatorname{sign}_{\sigma}(\phi(u)-\phi(k))(f(k)-D) . \eta \xi \leq \int_{0}^{T} \int_{\partial \Omega}|(f(k)-D) \cdot \eta(x)| \xi .
$$

Then, we obtain the required inequality (4.1).

Now, we consider the stationary problem associated to problem $(P)$ :

$$
(S)\left\{\begin{aligned}
u+\operatorname{div}(f(u)-\nabla \phi(u)) & =g \text { in } \Omega, \\
(f(u)-\nabla \phi(u)) \cdot \eta & =0 \text { on } \partial \Omega .
\end{aligned}\right.
$$

Remark 4.5. If $u(x)$ independent of $t$ is solution of $(S)$ then $u(t, x)=u(x)$ is solution of $(P)$ with the source term $g-u$. Then, we can deduce from Definition 2.3 and Proposition 4.2 their equivalent form for the stationary problem.

Definition 4.6. Let $g$ a measurable function taking values in $\left[0, u_{\max }\right]$. A measurable function $u$ taking values in $\left[0, u_{\text {max }}\right]$ is an entropy solution of $(S)$, if $\phi(u) \in H^{1}(\Omega)$ and for all $\xi \in \mathcal{C}^{\infty}\left(\mathbb{R}^{N}\right)^{+}, \forall k \in$ $\left[0, u_{\max }\right]$,

$$
\begin{aligned}
& -\int_{\Omega} \operatorname{sign}(u-k) u \xi d y+\int_{\Omega} \operatorname{sign}(u-k)[f(u)-f(k)-\nabla \phi(u)] . \nabla \xi d y \\
& +\int_{\partial \Omega}|f(k) \cdot \eta(y)| \xi d \mathcal{H}^{N-1}+\int_{\Omega} \operatorname{sign}(u-k) g \xi d y \geq 0 .
\end{aligned}
$$


Proposition 4.7. Let $\xi \in \mathcal{C}^{\infty}\left(\mathbb{R}^{N}\right)$; then for all $k \in\left[u_{c}, u_{\max }\right]$, for all $D \in \mathbb{R}^{N}$, for all entropy solution $u$ of $(S)$, we have:

$$
\begin{aligned}
& -\int_{\Omega} \operatorname{sign}(u-k) u \xi d y+\int_{\Omega} \operatorname{sign}(u-k)[f(u)-f(k)-\nabla \phi(u)+D] . \nabla \xi d y \\
& +\int_{\partial \Omega}|(f(k)-D) \cdot \eta(y)| \xi d \mathcal{H}^{N-1}+\int_{\Omega} \operatorname{sign}(u-k) g \xi d y \\
& \geq \lim _{\sigma \rightarrow 0} \frac{1}{\sigma} \int_{\Omega \cap\{-\sigma<\phi(u)-\phi(k)<\sigma\}} \nabla \phi(u)(\nabla \phi(u)-D) \xi .
\end{aligned}
$$

From now on, we will suppose that $\Omega=(a, b)$ is a bounded interval of $\mathbb{R}$.

Proposition 4.8. For all measurable function $g$ taking values in $\left[0, u_{\max }\right]$ the problem $(S)$ admits a solution $u$ such that $\left(f(u)-\phi(u)_{y}\right)$ is continuous up the boundary, i.e., $\left(f(u)-\phi(u)_{y}\right) \in \mathcal{C}([a, b])$. Moreover, $f(u)-\phi(u)_{y}$ is zero at $y=a$ and $y=b$.

Proof. For existence of entropy solution, we can refer to [16, Chap 2], using Galerkin approximations, in a way similar to Theorem 3.4 and 3.1.

Since $u$ is a weak solution of $(S)$, this means that $\left(f(u)-\phi(u)_{y}\right)_{y}=g-u$ in $\mathcal{D}^{\prime}$. Then $\left(f(u)-\phi(u)_{y}\right)_{y} \in$ $L^{\infty}([a, b])$, which implies that $\left(f(u)-\phi(u)_{y}\right) \in W^{1, \infty}([a, b]) \subset \mathcal{C}([a, b])$.

Now, as in Remarks 2.4, item 3, from (4.3) we deduce that $\left(f(u)-\phi(u)_{y}\right)_{\left.\right|_{\partial \Omega}}=0$ in the weak sense. Therefore $f(u)-\phi(u)_{y} \in \mathcal{C}_{0}([a, b])$.

To continue, we will recast problem $(P)$ under the abstract form of an evolution equation governed by an accretive operator, in order to apply classical results of the nonlinear semigroup theory (see, e.g., [6]). Let us define the (possibly multivalued) operator $A_{f, \phi}$ by it resolvent

$$
(u, z) \in A_{f, \phi}=\{u \text { such that } u \text { is an entropy solution of }(S) \text {, with } g=u+z\} .
$$

For an operator $A: L^{1}(\Omega) \rightarrow L^{1}(\Omega)$, denote by $R(A)$ its range, by $D(A)$ its domain and by $\overline{R(A)}$, $\overline{D(A)}$ their closures in $L^{1}(\Omega)$ respectively.

Let us stress that for $u \in D(A), f(u)-\phi(u)_{y} \in \mathcal{C}_{0}([a, b])$ due to Proposition 4.8.

Recall (cf. [6]) that an operator $A$ is accretive if $[\beta-\hat{\beta}, \alpha-\hat{\alpha}]_{L^{1}(\Omega)} \geq 0$ for all $(\beta, \alpha),(\hat{\beta}, \hat{\alpha}) \in A$, where for $\beta, \alpha \in L^{1}(\Omega)$ the bracket $[.,]_{L^{1}(\Omega)}$ is defined by $[\beta, \alpha]_{L^{1}(\Omega)}=\int_{\Omega} \operatorname{sign}(\beta) \alpha+\int_{[\beta=0]}|\alpha|$. If $A$ is accretive and $R(I+\lambda A)=L^{1}(\Omega)$ for some $\lambda>0$, then $A$ is m-accretive.

Proposition 4.9. Let $(u, z) \in A_{f, \phi},(\hat{u}, \hat{z}) \in A_{f, \phi}$. Then for $\xi \in \mathcal{C}^{\infty}(\bar{\Omega})^{+}$

$$
\begin{aligned}
& \int_{\Omega}|u-\hat{u}| \xi d y+\int_{\Omega} \operatorname{sign}(u-\hat{u})\left[f(u)-f(\hat{u})-\phi(u)_{y}+\phi(\hat{u})_{y}\right] \cdot \xi_{y} d y \\
& \leq \int_{\Omega} \operatorname{sign}(u-\hat{u})(g-\hat{g}) \xi d y+\int_{[u=\hat{u}]}|g-\hat{g}| \xi d y=[u-\hat{u}, g-\hat{g}]_{L^{1}(\Omega)} .
\end{aligned}
$$

Proof. (Sketched) The proof of Proposition 4.5 is actually contained in the proof of Theorem 4.13 below, due to Remark 2.4. Actually a simpler argument applies, because both $f(\hat{u})-\phi(\hat{u})_{y}$ and $f(u)-\phi(u)_{y}$ have strong trace in the context of the stationary problem $(S)$.

Somewhat abusively, we will write $L^{1}\left(\Omega ;\left[0, u_{\max }\right]\right)$ for the set of all mesurable functions from $[a, b]$ to $\left[0, u_{\max }\right]$.

Proposition 4.10. 1. $A_{f, \phi}$ is accretive in $L^{1}(\Omega)$.

2. For all $\lambda$ sufficiently small, $R\left(I+\lambda A_{f, \phi}\right)$ contains $L^{1}\left(\Omega ;\left[0, u_{\max }\right]\right)$.

3. $\overline{D\left(A_{f, \phi}\right)}=L^{1}\left(\Omega ;\left[0, u_{\max }\right]\right)$. 
Proof. 1. Let $(u, z) \in A_{f, \phi},(\hat{u}, \hat{z}) \in A_{f, \phi}$. Applying Proposition 4.9 with $\xi=1$ in (4.5) and the standard properties of the bracket (see [6]), we get

$$
\begin{aligned}
\|u-\hat{u}\|_{L^{1}(\Omega)} & \leq[u-\hat{u}, g-\hat{g}]_{L^{1}(\Omega)} \\
& \leq[u-\hat{u}, u-\hat{u}+z-\hat{z}]_{L^{1}(\Omega)} \\
& \leq\|u-\hat{u}\|_{L^{1}(\Omega)}+[u-\hat{u}, z-\hat{z}]_{L^{1}(\Omega)} .
\end{aligned}
$$

We deduce that $[u-\hat{u}, z-\hat{z}]_{L^{1}(\Omega)} \geq 0$, so that $A_{f, \phi}$ is accretive.

2. For $\lambda>0$, consider the problem

$$
\left(S_{\lambda}\right)\left\{\begin{aligned}
u_{\lambda}+\lambda\left(f\left(u_{\lambda}\right)-\left(\phi\left(u_{\lambda}\right)\right)_{y}\right)_{y} & =g \text { in } \Omega \\
\lambda\left(f\left(u_{\lambda}\right)-\phi\left(u_{\lambda}\right)_{y}\right) \cdot \eta(y) & =0 \text { on } \partial \Omega .
\end{aligned}\right.
$$

Notice that the notion of solution for $\left(S_{\lambda}\right)$ is like the Definition 4.6. Let $g \in L^{1}\left(\Omega ;\left[0, u_{\max }\right]\right)$, and $\lambda>0$ then, there exists $u_{\lambda}$ entropy solution of $\left(S_{\lambda}\right)$ (see Proposition 4.8) such that $\left(u_{\lambda}, \frac{g-u_{\lambda}}{\lambda}\right) \in A_{f, \phi}$. Hence $g \in R\left(I+\lambda A_{f, \phi}\right)$ and therefore $R\left(I+\lambda A_{f, \phi}\right) \supset L^{1}\left(\Omega ;\left[0, u_{\max }\right]\right)$, which was to be shown.

3 . Let $P C\left([a, b] ;\left[0, u_{\max }\right]\right)$ be the set of piecewise constant functions from $[a, b]$ to $\left[0, u_{\max }\right]$. Then $P C\left([a, b] ;\left[0, u_{\max }\right]\right)$ is dense in $L^{1}\left([a, b] ;\left[0, u_{\max }\right]\right)$. Take $g \in P C\left([a, b] ;\left[0, u_{\max }\right]\right), g=\sum_{i} c_{i} \mathbf{1}_{\left(a_{i}, b_{i}\right)}$ where the $\left(a_{i}, b_{i}\right)$ are disjoint intervals. There exists $u_{n} \in L^{\infty}(a, b)$ entropy solution of $\left(S_{\frac{1}{n}}\right)$, i.e, we have $\left(u_{n}, n\left(g-u_{n}\right)\right) \in A_{f, \phi}$. For $k \in\left[0, u_{\max }\right]$, for all $\xi \in \mathcal{C}_{0}^{\infty}(\mathbb{R})$ we get

$$
\begin{aligned}
& \frac{1}{n} \int_{a}^{b} \operatorname{sign}\left(u_{n}-k\right)\left(f\left(u_{n}\right)-f(k)-\partial_{y} \phi\left(u_{n}\right)\right) \cdot \partial_{y} \xi d y \\
& +\int_{a}^{b} \operatorname{sign}\left(u_{n}-k\right)\left(g-u_{n}\right) \xi d y+\frac{1}{n} \int_{a, b}|f(k) \cdot \eta(y)| \xi d \sigma \geq 0 .
\end{aligned}
$$

For every $i$, one can construct $\xi_{i}^{n}$ such that $\xi_{i}^{n} \rightarrow \mathbf{1}_{\left(a_{i}, b_{i}\right)}$, as $n \rightarrow \infty, \operatorname{supp}_{i}^{n} \subset\left(a_{i}, b_{i}\right),\left\|\partial_{y} \xi_{i}^{n}\right\|_{L^{\infty}} \leq$ $2 \sqrt[3]{n}$ and $\xi_{i}^{n} \equiv 1$ in $\left(a_{i}+\delta_{n}^{i}, b_{i}-\delta_{n}^{i}\right)$ with $\delta_{n}^{i}=\frac{b_{i}-a_{i}}{2 \sqrt[3]{n}}$.

Take $k=c_{i}$ and $\xi=\xi_{i}^{n}$ in (4.6).

$$
\begin{aligned}
\int_{a_{i}+\delta_{n}^{i}}^{b_{i}-\delta_{n}^{i}}\left|u_{n}-c_{i}\right| d y & \left.\leq \frac{1}{n} \int_{a_{i}+\delta_{n}^{i}}^{b_{i}-\delta_{n}^{i}} \operatorname{sign}\left(u_{n}-c_{i}\right)\left(f\left(u_{n}\right)-f\left(c_{i}\right)-\partial_{y} \phi\left(u_{n}\right)\right)\right) \partial_{y} \xi_{i}^{n} d y \\
& \leq \frac{2}{n}|b-a|\|f\|_{L^{\infty}}|.| \partial_{y} \xi_{i}^{n}\left\|_{L^{1}}+\right\| \frac{1}{\sqrt{n}} \partial_{y} \phi\left(u_{n}\right)\left\|_{L^{2}} .\right\| \partial_{y} \xi_{i}^{n} \|_{L^{2}} .
\end{aligned}
$$

Then, for all $\delta>\delta_{n}^{i}, u_{n} \rightarrow g$ a.e on $\cup_{i}\left(a_{i}+\delta, b_{i}-\delta\right)$. We conclude by the Lebesgue theorem that $u_{n} \rightarrow g$ in $L^{1}([a, b])$.

In conclusion, $D\left(A_{f, \phi}\right)$ is dense in $P C\left([a, b] ;\left[0, u_{\max }\right]\right)$ and therefore, it is also dense in $L^{1}\left(\Omega ;\left[0, u_{\max }\right]\right)$.

Now, we can exploit the notion of integral solution (see, e.g., $[6,7]$ ).

Definition 4.11. Suppose that $h \in L^{1}(Q), u_{0} \in L^{1}(\Omega)$. A function $v \in \mathcal{C}\left([0, T] ; L^{1}\left([a, b] ;\left[0, u_{\max }\right]\right)\right)$ is an integral solution of the problem

$$
v_{t}+A_{f, \phi}(v) \ni h, \quad v(t=0)=u_{0},
$$

if $v(0,)=.u_{0}($.$) and for all (u, z) \in A_{f, \phi}$

$$
\frac{d}{d t}\|v(t)-u\|_{L^{1}(\Omega)} \leq[v(t)-u, h(t)-z]_{L^{1}(\Omega)} \text { in } \mathcal{D}^{\prime}(0, T) .
$$

By Proposition 4.10 , the operator $A_{f, \phi}$ is m-accretive ${ }^{1}$ densely defined in $L^{1}\left(\Omega ;\left[0, u_{\text {max }}\right]\right)$, by the general theory of non-linear semigroups (cf. $[6,7,8]$ ), we have the following result.

${ }^{1}$ Rigourously speaking, this statement is false because $L^{1}\left(\Omega ;\left[0, u_{\max }\right]\right)$ is not a Banach space, but its convex subset. Nonetheless, this subset is invariant for the stationary problem (S), therefore the nonlinear semigroups theory applies without change in our case. 
Corollary 4.12. Let $\Omega=(a, b), u_{0}, \hat{u}_{0} \in L^{1}(\Omega)$ and $h, \hat{h} \in L^{1}(Q)$. Let $v, \hat{v}$ be integral solutions of (4.7) associated with the data $\left(u_{0}, h\right)$ and $\left(\hat{u}_{0}, \hat{h}\right)$, respectively. Then for a.e. $t \in[0, T)$.

$$
\|v(t)-\hat{v}(t)\|_{L^{1}} \leq\left\|u_{0}-\hat{u}_{0}\right\|_{L^{1}}+\int_{0}^{t}\|h(\tau)-\hat{h}(\tau)\|_{L^{1}} d t
$$

In particular, the integral solution is unique.

Theorem 4.13. Let $\Omega=[a, b]$. Let $v$ be an entropy solution of $(P)$ and $u$ be an entropy solution of $(S)$. Then

$$
\frac{d}{d t}\|v(t)-u\|_{L^{1}(\Omega)} \leq \int_{\Omega} \operatorname{sign}(v-u)(u-g) d x \text { in } \mathcal{D}^{\prime}(0, T)
$$

In particular, $v$ is an integral solution of (4.7) with $h=0$.

First, note the following auxiliary result.

Lemma 4.14. ([2]) Let $\delta$ be a positive function with support in $[-1,1]$ and $\|\delta\|_{L^{1}}=1$. Assume that for all $z \in[-1,1], w_{n}(., z) \rightarrow w($.$) and h_{n}(., z) \rightarrow h($.$) in L^{1}(\mathbb{R})$ as $n \rightarrow \infty$. If in addition $\left\|h_{n}(., z)\right\|_{L^{1}(\mathbb{R})}$ is bounded uniformly in $n$ and $z$, then the below limit exists and the following equality holds:

$$
\limsup _{n \rightarrow \infty} \iint \operatorname{signw}_{n}(x, z) h_{n}(x, z) \delta(z) \leq[w, h]
$$

Moreover, if for all $n \in \mathbb{N}$ and a.e. $z \in[-1,1], h_{n}(., z)=0$ a.e. on $\left\{w_{n}(., z)=0\right\}$ and if $h=0$ a.e. on $\{w=0\}$, then there exists

$$
\lim _{n \rightarrow \infty} \iint \operatorname{signw}_{n}(x, z) h_{n}(x, z) \delta(z)=\int \operatorname{sign}(w) h .
$$

Proof. The claim of Inequality (4.9) follows from the definition and the upper semicontinuity of the bracket, the definition of $\delta$ and the Fatou lemma. Inequality (4.10) follows by applying the first one to $w_{n}, h_{n}$ and to $-w_{n}, h_{n}$.

Proof of Theorem 4.13. To start with, note that by the result of [12] an entropy solution $v$ of $(P)$ is automaticaly time-continuous with values in $L^{1}\left(\Omega ;\left[0, u_{\max }\right]\right)$.

Now, we apply the doubling of variables [15] in the way of [2]. We consider $v=v(t, x)$ an entropy solution of $(P)$ and $u=u(y)$ an entropy solution of $(S)$. Consider nonnegative function $\xi=\xi(t, x, y)$ having the property that $\xi(., ., y) \in \mathcal{C}^{\infty}([0, T) \times \bar{\Omega})$ for each $y \in \bar{\Omega}, \xi(t, x,.) \in \mathcal{C}_{0}^{\infty}(\bar{\Omega})$ for each $(t, x) \in[0, T) \times \bar{\Omega}$.

We denote $\Omega_{x}=\left\{x \in \Omega ; v(t, x) \in\left[0, u_{c}\right]\right\} ; \Omega_{y}=\left\{y \in \Omega ; u(y) \in\left[0, u_{c}\right]\right\}$ and $\Omega_{x}^{c}, \Omega_{y}^{c}$ their complementaries in $\Omega$. In (4.1), take $\xi=\xi(t, x, y), k=u(y), D=\phi(u)_{y}$ and integrate over $\Omega_{y}^{c}$. We get

$$
\begin{aligned}
& \int_{\Omega_{y}^{c}} \int_{0}^{T} \int_{x \in \Omega}\left\{|v-u| \xi_{t}+\operatorname{sign}(v-u)\left[f(v)-\phi(v)_{x}-f(u)+\phi(u)_{y}\right] \cdot \xi_{y}\right\} \\
& +\int_{\Omega_{y}^{c}} \int_{0}^{T} \int_{x \in \partial \Omega}\left|\left(f(u)-\phi(u)_{y}\right) \cdot \eta(x)\right| \xi d \sigma d t d y+\int_{\Omega_{y}^{c}} \int_{x \in \Omega}\left|v_{0}-u\right| \xi(0, x, y) \\
& \geq \lim _{\sigma \rightarrow 0} \frac{1}{\sigma} \int_{\Omega_{y}^{c}} \int_{0}^{T} \int_{x \in \Omega \cap\{-\sigma<\phi(v)-\phi(u)<\sigma\}} \phi(v)_{x}\left(\phi(v)_{x}-\phi(u)_{y}\right) \xi .
\end{aligned}
$$

In the same way, in (2.2) take $\xi=\xi(t, x, y), k=u(y)$, integrate over $\Omega_{y}$, and use the fact that $\phi(u)_{y}=0$ in $\Omega_{y}$. We get

$$
\begin{aligned}
& \int_{\Omega_{y}} \int_{0}^{T} \int_{x \in \Omega}\left\{|v-u| \xi_{t}+\operatorname{sign}(v-u)\left[f(v)-\phi(v)_{x}-f(u)+\phi(u)_{y}\right] \cdot \xi_{y}\right\} \\
& +\int_{\Omega_{y}} \int_{0}^{T} \int_{x \in \partial \Omega}\left|\left(f(u)-\phi(u)_{y}\right) \cdot \eta(x)\right| \xi+\int_{\Omega_{y}} \int_{x \in \Omega}\left|v_{0}-u\right| \xi(0, x, y) \geq 0
\end{aligned}
$$


Since $\Omega=\Omega_{x} \cup \Omega_{x}^{c}$, by adding (4.11) to (4.12) we obtain:

$$
\begin{aligned}
& \int_{\Omega} \int_{0}^{T} \int_{\Omega}\left\{|v-u| \xi_{t}+\operatorname{sign}(v-u)\left[f(v)-\phi(v)_{x}-f(u)+\phi(u)_{y}\right] \cdot \xi_{x}\right\} \\
& +\int_{\Omega} \int_{0}^{T} \int_{x \in \partial \Omega}\left|\left(f(u)-\phi(u)_{y}\right) \cdot \eta(x)\right| \xi+\int_{\Omega} \int_{\Omega}\left|v_{0}-u\right| \xi(0, x, y) \\
& \geq \lim _{\sigma \rightarrow 0} \frac{1}{\sigma} \int_{\Omega_{y}^{c}} \int_{0}^{T} \int_{x \in \Omega \cap\{-\sigma<\phi(v)-\phi(u)<\sigma\}} \phi(v)_{x}\left(\phi(v)_{x}-\phi(u)_{y}\right) \xi .
\end{aligned}
$$

We proceed in the same way, exchanging the roles of $v$ and $u$. Starting from (4.4) and (4.3), we deduce

$$
\begin{aligned}
& \int_{0}^{T} \int_{\Omega} \int_{\Omega} \operatorname{sign}(v-u)\left[f(v)-\phi(v)_{x}-f(u)+\phi(u)_{y}\right] \cdot \xi_{y} d y d x d t \\
& +\int_{0}^{T} \int_{\Omega} \int_{y \in \partial \Omega}\left|\left(f(v)-\phi(v)_{x}\right) \cdot \eta(y)\right| \xi d \sigma d x d t \\
& +\int_{0}^{T} \int_{\Omega} \int_{\Omega} \operatorname{sign}(v-u)(u-g(y)) \xi d x d t d y \\
& \geq \lim _{\sigma \rightarrow 0} \frac{1}{\sigma} \int_{\Omega_{x}^{c}} \int_{0}^{T} \int_{y \in \cap\{-\sigma<\phi(u)-\phi(v)<\sigma\}} \phi(u)_{y}\left(\phi(u)_{y}-\phi(v)_{x}\right) \xi .
\end{aligned}
$$

Now, sum (4.13) and (4.14) to obtain

$$
\begin{aligned}
& \int_{0}^{T} \int_{\Omega} \int_{\Omega}|v-u| \xi_{t} d y d x d t+\int_{\Omega} \int_{\Omega}\left|v_{0}-u\right| \xi(0, x, y) d x d y \\
& +\int_{0}^{T} \int_{\Omega} \int_{\Omega} \operatorname{sign}(v-u)\left[\left(f(v)-\phi(v)_{x}\right)-\left(f(u)+\phi(u)_{y}\right)\right] \cdot\left(\xi_{x}+\xi_{y}\right) \\
& +\int_{0}^{T} \int_{x \in \partial \Omega} \int_{\Omega}\left|\left(f(u)-\phi(u)_{y}\right) \cdot \eta(x)\right| \xi d \sigma d t d y \\
& +\int_{0}^{T} \int_{\Omega} \int_{y \in \partial \Omega}\left|\left(f(v)-\phi(v)_{x}\right) \cdot \eta(y)\right| \xi d y d \sigma d t \\
& +\int_{0}^{T} \int_{\Omega} \int_{\Omega} \operatorname{sign}(v-u)(u-g(y)) \xi \\
& \geq \lim _{\sigma \rightarrow 0} \frac{1}{\sigma} \int_{0}^{T} \iint_{\Omega_{x}^{c} \times \Omega_{y}^{c} \cap\{-\sigma<\phi(v)-\phi(u)<\sigma\}}\left|\phi(v)_{x}-\phi(u)_{y}\right|^{2} \xi d y d x d t \geq 0 .
\end{aligned}
$$

Next, following the idea of [2] we consider the test function $\xi(t, x, y)=\theta(t) \rho_{n}(x, y)$, where $\theta \in \mathcal{C}_{0}^{\infty}(0, T)$, $\theta \geq 0, \rho_{n}(x, y)=\delta_{n}(\Delta)$ and $\Delta=\left(1-\frac{1}{n(b-a)}\right) x-y+\frac{a+b}{2 n(b-a)}$. Then, $\rho_{n} \in \mathcal{D}(\bar{\Omega} \times \bar{\Omega})$ and $\rho_{n_{\mid \Omega \times \partial \Omega}}(x, y)=0$.

Due to this choice

$$
\int_{0}^{T} \int_{x \in \Omega} \int_{y \in \partial \Omega}\left|\left(f(v)-\phi(v)_{x}\right) \cdot \eta(y)\right| \rho_{n} \theta d y d \sigma d t=0 .
$$

By Proposition 4.8, $\left(f(u)-\phi(u)_{y}\right) \in \mathcal{C}_{0}([a, b])$. Therefore we have $\left|\left(f(u)-\phi(u)_{y}\right) \cdot \eta(x)\right| \longrightarrow 0$ when $x \rightarrow y$, i.e, as $n \longrightarrow \infty$. We conclude that

$$
\lim _{n \rightarrow \infty} \int_{0}^{T} \int_{x \in \partial \Omega} \int_{y \in \Omega}\left|\left(f(u)-\phi(u)_{y}\right) \cdot \eta(x)\right| \rho_{n} \theta d y d \sigma d t=0 .
$$

It remains to study the limit, as $n \rightarrow \infty$

$$
I_{n}=\int_{0}^{T} \int_{\Omega} \int_{\Omega} \theta \operatorname{sign}(v-u)\left[\left(f(v)-\phi(v)_{x}\right)-\left(f(u)-\phi(u)_{y}\right)\right] \cdot\left(\left(\rho_{n}\right)_{x}+\left(\rho_{n}\right)_{y}\right) d y d x d t .
$$


We use the change of variable $(x, y) \mapsto(x, z)$ with $z=n(x-y)-\frac{1}{b-a} x+\frac{a+b}{b-a}$,

$$
\begin{aligned}
I_{n} & =\frac{2}{b-a} \int_{-1}^{1} \int_{0}^{T} \int_{\Omega} \operatorname{sign}(v-u)\left[\left(f(v)-\phi(v)_{x}\right)-\left(f(u)-\phi(u)_{y}\right)\right] \cdot \delta_{n}^{\prime}(z) \theta \\
& =\frac{2}{b-a} \int_{-1}^{1} \int_{0}^{T} \int_{a}^{b} \operatorname{sign}\left(v(t, x)-u_{n}(x, z)\right)\left[p(t, x)-q_{n}(x, z)\right] \delta_{n}^{\prime}(z) \theta(t),
\end{aligned}
$$

where $u_{n}(x, z):=u(y), p(t, x):=f(v)-\phi(v)_{x}$ and $q_{n}:=f(u)-\phi(u)_{y}$. For $z$ given, $u_{n}(., z)$ converges to $u($.$) in L^{1}$ and $q_{n}(., z)$ converges to $q():.=f(u)-\phi(u)_{x}$ in $L^{1}$. From Lemma 4.14, we deduce that for all $z \in[-1,1]$

$$
K_{n}(z):=\int_{Q} \operatorname{sign}\left(v_{n}(t, x, z)\right) h_{n}(t, x, z) d x d t \longrightarrow_{n \rightarrow \infty} \int_{Q} \operatorname{sign}(v) h d x d t=: K=\text { const },
$$

where $v_{n}:=v-u_{n}, h_{n}:=p-q_{n}$ and $h:=p-q$. Then $K_{n}($.$) converges to K$ independently of $z$. Moreover, from the definition of $K_{n}$ one finds easily the uniform $L^{\infty}$ bound $\left|K_{n}\right| \leq 2\left(\|p\|_{L^{1}(Q)}+\right.$ $\left.T\|q\|_{L^{1}(\Omega)}\right)$, for $n$ large enough. Hence by the Lebesgue theorem,

$$
\lim _{n \rightarrow \infty} \int_{-1}^{1} K_{n}(z) \delta^{\prime}(z)=K \int_{-1}^{1} \delta^{\prime}(z)=0 .
$$

We have shown that the limit of $I_{n}$ equals zero. The passage to the limit in other terms in (4.15) is straightforward. Finally (4.15) gives for $n \longrightarrow \infty$

$$
\int_{0}^{T} \int_{\Omega}|v(t, x)-u(y)| \theta^{\prime}(t) d x d t+\int_{0}^{T} \int_{\Omega} \operatorname{sign}(v-u)(u-g) \theta \geq 0 .
$$

Hence

$$
\frac{d}{d t}\|v(t)-u\|_{L^{1}(\Omega)} \leq \int_{\Omega} \operatorname{sign}(v-u)(u-g) d x \text { in } \mathcal{D}^{\prime}(0, T) .
$$

Thus, $v$ is an integral solution of (4.7).

Now, the claim of Theorem 4.1 is a direct consequence of the fact that the entropy solution is also an integral solution, and of Corollary 4.12 .

\section{Appendix: Existence of entropy solutions for the viscosity regularized problem}

For the sake of completeness, we give a full proof of Theorem 3.4. We denote by $C$ a generic constant independent of the approximation parameters $\epsilon$ and $m$. Otherwise, the dependence of $C$ is made explicit in the notation.

Proof of Theorem 3.4. We need four steps for this proof.

First step: By Faedo-Galerkin method (see e.g., [16]), we construct a sequence of approximate solutions. We choose $V_{m}=\left\langle e_{1}(x), \ldots, e_{m}(x)\right\rangle$ with $\left(e_{i}\right)_{i=1}^{\infty}$ a regular Hilbert basis of $H^{1}(\Omega)$ and formulate our problem in terms of the new unknown $w^{\epsilon}=\phi_{\epsilon}\left(u^{\epsilon}\right)$. We seek $w_{m}^{\epsilon}(t)=\sum_{i=1}^{m} c_{i m}(t) e_{i}(x)$, then

$$
\operatorname{Proj}_{V_{m}}\left(\phi_{\epsilon}^{-1}\left(w_{m}^{\epsilon}\right)_{t}+\operatorname{div} f\left(\phi_{\epsilon}^{-1}\left(w_{m}^{\epsilon}\right)\right)-\Delta w_{m}^{\epsilon}\right)=0 .
$$

Here $\operatorname{Proj}_{V_{m}}$ is the orthogonal projection, in $L^{2}(\Omega)$, on the subspace $V_{m}$. The function $\phi_{\epsilon}^{-1}$ is Lipschitz continuous, and $\left(\phi_{\epsilon}^{-1}\right)^{\prime} \leq \frac{1}{\epsilon}$. To start with, we assume that $\phi$ is Lipschitz continuous; then $\left(\phi_{\epsilon}^{-1}\right)^{\prime} \geq$ $\alpha>0$. The equation 5.1 is rewrites as

$$
\operatorname{Proj}_{V_{m}}\left(\left(\phi_{\epsilon}^{-1}\right)^{\prime}\left(w_{m}^{\epsilon}\right) \sum_{i=1}^{m} c_{i m}^{\prime} e_{i}(x)+\operatorname{div} \tilde{f}\left(w_{m}^{\epsilon}\right)-\sum_{i=1}^{m} c_{i m}(t) \Delta e_{i}(x)\right)=0
$$


where $\tilde{f}=f \circ \phi_{\epsilon}^{-1}$. To determine the family $\left\{c_{i m}\right\}_{i} \subset \mathcal{C}^{1}([0, T])$, we write the weak formulation of the above equation in $\Omega$ with $e_{j}$ as test function, we get

$$
\sum_{i=1}^{m} c_{i m}^{\prime}(t) \int_{\Omega}\left(\phi_{\epsilon}^{-1}\right)^{\prime}\left(w_{m}^{\epsilon}\right) e_{i} \cdot e_{j} d x-\int_{\Omega}\left(\tilde{f}\left(w_{m}^{\epsilon}\right)-\sum_{i=1}^{m} c_{i m} \nabla e_{i}(x)\right) \cdot \nabla e_{j} d x=0
$$

$1 \leq j \leq m$. Recall that $w_{m}^{\epsilon}$ depends on $x$ and $\left(c_{i m}\right)_{i}$. Notice that the matrix $M\left(c_{i 1}, \ldots, c_{i m}\right)=$ $\left(\int_{\Omega}\left(\phi_{\epsilon}^{-1}\right)^{\prime}\left(w_{m}^{\epsilon}\right) e_{i} . e_{j} d x\right)_{i, j}$ is invertible due to the fact that for all $b=\left(b_{1}, \ldots, b_{m}\right) \in \mathbb{R}^{m}$,

$$
(M b, b)=\int_{\Omega}\left(\phi_{\epsilon}^{-1}\right)^{\prime}\left(w_{m}^{\epsilon}\right)\left|\sum_{i=1}^{m} b_{i} e_{i}\right|^{2} \geq \operatorname{const}(m) \alpha\|b\|^{2} .
$$

We obtain a system of non-linear differential equations, which is completed with initial condition $w_{m}^{\epsilon}(0)=w_{0 m}^{\epsilon} ; w_{0 m}^{\epsilon}=\sum_{i=1}^{m} \beta_{i m} e_{i}$ where $\beta_{i m}$ are chosen to ensure that $w_{0 m}^{\epsilon} \longrightarrow \phi_{\epsilon}\left(u_{0}^{\epsilon}\right)$ in $L^{2}(\Omega)$.

By the Cauchy-Peano theorem of the classical ODE theory, we have existence of solution $w_{m}^{\epsilon}(t)$ in some interval $\left[0, t_{m}\right], t_{m}>0$. Note that existence of $u_{m}^{\epsilon}$ is ensured by the fact that $\phi_{\epsilon}$ is bijective, moreover, $w_{m}^{\epsilon}=\phi_{\epsilon}\left(u_{\epsilon}^{m}\right)$ and $u_{m}^{\epsilon}$ is in $\mathcal{C}^{1}\left(0, t_{m} ; V_{m}\right)$.

Now, we have to prove that $t_{m}=T$.

Second step: a priori estimates.

We can take $w_{m}^{\epsilon}$ as a test function and integrate over $[0, t]$; we get

$$
\int_{\Omega} \int_{0}^{t} \theta_{\epsilon}\left(w_{m}^{\epsilon}\right)_{s} d s d x-\int_{0}^{t} \int_{\Omega} f\left(u_{m}^{\epsilon}\right) \cdot \nabla w_{m}^{\epsilon} d x d s+\left\|\nabla w_{m}^{\epsilon}\right\|_{L^{2}(0, t ; \Omega)}^{2}=0 .
$$

Here $\theta_{\epsilon}\left(w_{m}^{\epsilon}\right)=\int_{0}^{w_{m}^{\epsilon}} r d \phi_{\epsilon}^{-1}(r)$ and we have used the chain rule for $\mathcal{C}^{1}$ functions of variable $t$. It is also possible to rewrite the function $\theta_{\epsilon}\left(w_{m}^{\epsilon}\right)$ as $\psi_{\epsilon}\left(u_{m}^{\epsilon}\right)=\int_{0}^{u_{m}^{\epsilon}} \phi_{\epsilon}(r) d r$. Since $f$ has its support in $\left[0, u_{\max }\right]$, the second integral of (5.2) can be upper bounded as follows

$$
\begin{aligned}
\left|\int_{0}^{t} \int_{\Omega} \operatorname{div}\left(\int_{0}^{u_{m}^{\epsilon}} f(r) d \phi_{\epsilon}(r)\right) d x d s\right| & =\left|\int_{0}^{t} \int_{\partial \Omega}\left(\int_{0}^{u_{m}^{\epsilon}} f(r) d \phi_{\epsilon}(r)\right) \cdot \eta d \mathcal{H}^{N-1} d s\right| \\
& \leq d \phi_{\epsilon}\left(\left[0, u_{\max }\right]\right)\|f\|_{L^{\infty}}|\partial \Omega| T \\
& \leq\left(\phi\left(u_{\max }\right)+1\right)\|f\|_{L^{\infty}}|\partial \Omega| T . \\
& \leq C=C\left(T, \partial \Omega,\|f\|_{L^{\infty}}\right),
\end{aligned}
$$

with $d \phi_{\epsilon}\left(\left[0, u_{\max }\right]\right)$ the measure of $\left[0, u_{\max }\right]$ with respect to the Stieltjes measure $d \phi_{\epsilon}$. Hence,

$$
\int_{\Omega} \theta_{\epsilon}\left(w_{m}^{\epsilon}\right)(t) d x+\left\|\nabla w_{m}^{\epsilon}\right\|_{L^{2}(0, t ; \Omega)}^{2} \leq C+\int_{\Omega} \theta_{\epsilon}\left(w_{m}^{\epsilon}\right)(0) d x .
$$

The last term in the right-hand side of inequality (5.3) is bounded uniformly in $m$ by $\frac{1}{\epsilon} \sup _{m}\left\|w_{0 m}^{\epsilon}\right\|_{L^{2}}^{2}$. In fact,

$$
\int_{\Omega} \theta_{\epsilon}\left(w_{m}^{\epsilon}\right)(0) d x=\int_{\Omega} \int_{0}^{w_{m}^{\epsilon}(0)} r\left(\phi_{\epsilon}^{-1}\right)^{\prime}(\sigma) d r d x \leq \frac{1}{2}\left\|\left(\phi_{\epsilon}^{-1}\right)^{\prime}\right\|_{\infty}\left\|w_{m}^{\epsilon}\right\|_{L^{2}(\Omega)}^{2} \leq \frac{1}{\epsilon} \sup _{m}\left\|w_{0 m}^{\epsilon}\right\|_{L^{2}(\Omega)}^{2} .
$$

Then $\nabla w_{m}^{\epsilon}$ is bounded in $L^{2}(\Omega)$ uniformly in $m$.

Without loss of restriction, we can assume $\phi \equiv 0$ on $(-\infty, 0]$ and $\phi \equiv \phi\left(u_{\max }\right)$ on $\left[u_{\max },+\infty\right)$. (Indeed, we show in the last step that $u$ takes values in $\left[0, u_{\max }\right]$, therefore the values of $\phi$ outside $\left[0, u_{\max }\right]$ do not matter.) Then $\phi_{\epsilon}^{\prime}=\epsilon$ outside $\left[0, u_{\max }\right]$. Hence, for $w \notin\left[0, \phi\left(u_{\max }\right)+1\right]$, we have $\left(\phi_{\epsilon}^{-1}\right)^{\prime}(w)=\frac{1}{\epsilon}$. Therefore

$$
\left|w_{m}^{\epsilon}\right|^{2} \leq C(\epsilon)\left(1+\theta_{\epsilon}\left(w_{m}^{\epsilon}\right)\right)
$$


This means that $t_{m}=T$ and $w_{m}^{\epsilon}$ is bounded in $L^{2}\left(0, T, H^{1}(\Omega)\right)$ uniformly in $m$.

Now, fix $\delta$ and consider $t$ such that $[t, t+\delta] \subset[0, T]$. We integrate over $s \in[t, t+\delta t]$. Next, we take (by approximation) $\left(w_{m}^{\epsilon}(t+\delta t,)-.w_{m}^{\epsilon}(t,).\right) \mathbf{1}_{[0, T-\delta t]}$ as test function

$$
\begin{aligned}
& \int_{0}^{T-\delta t} \int_{t}^{t+\delta t} \int_{\Omega}\left(u_{m}^{\epsilon}(s, .)\right)^{\prime}\left(w_{m}^{\epsilon}(t+\delta t, .)-w_{m}^{\epsilon}(t, .)\right) d x d t d s \\
& -\int_{0}^{T-\delta t} \int_{t}^{t+\delta t} \int_{\Omega} f\left(u_{m}^{\epsilon}\right) \cdot \nabla\left(w_{m}^{\epsilon}(t+\delta t, .)-w_{m}^{\epsilon}(t, .)\right) d x d s d t \\
& +\int_{0}^{T-\delta t} \int_{t}^{t+\delta t} \int_{\Omega} \nabla w_{m}^{\epsilon}(s, x) \cdot \nabla\left(w_{m}^{\epsilon}(t+\delta t, .)-w_{m}^{\epsilon}(t, .)\right) d x d t d s=0 .
\end{aligned}
$$

Denote the three terms in the left-hand side of (5.5) by $A, B$ and $D$ respectively. We calculate

$$
\begin{aligned}
A & =\int_{0}^{T-\delta t} \int_{t}^{t+\delta t} \int_{\Omega}\left(u_{m}^{\epsilon}(s, .)\right)^{\prime}\left(w_{m}^{\epsilon}(t+\delta t, .)-w_{m}^{\epsilon}(t, .)\right) d x d t d s \\
& =\int_{0}^{T-\delta t} \int_{\Omega}\left(w_{m}^{\epsilon}(t+\delta t, .)-w_{m}^{\epsilon}(t, .)\right) \int_{t}^{t+\delta t}\left(u_{m}^{\epsilon}(s, .)\right)^{\prime} d s d x d t \\
& =\int_{0}^{T-\delta t} \int_{\Omega}\left(w_{m}^{\epsilon}(t+\delta t, .)-w_{m}^{\epsilon}(t, .)\right)\left(u_{m}^{\epsilon}(t+\delta t, .)-u_{m}^{\epsilon}(t, .)\right) d x d t \\
|B| & =\left|-\int_{0}^{T-\delta t} \int_{t}^{t+\delta t} \int_{\Omega} f\left(u_{m}^{\epsilon}(s, .)\right) . \nabla\left(w_{m}^{\epsilon}(t+\delta t, .)-w_{m}^{\epsilon}(t, .)\right) d x d t d s\right| \\
& \leq\left|\int_{0}^{T-\delta t} \int_{t}^{t+\delta t} \int_{\Omega} f\left(u_{m}^{\epsilon}(s, .)\right) . \nabla\left(w_{m}^{\epsilon}(t+\delta t, .)-w_{m}^{\epsilon}(t, .)\right) d x d t d s\right| \\
& \leq C(\epsilon) \delta t\left[\int_{0}^{T-\delta t} \int_{\Omega}\left|\nabla w_{m}^{\epsilon}(t+\delta t, .)\right|+\int_{0}^{T-\delta t} \int_{\Omega}\left|\nabla w_{m}^{\epsilon}(t, .)\right|\right] .
\end{aligned}
$$

By a change of variable in the first integral $(\tau=t+\delta t)$, using Cauchy-Schwarz inequality we obtain

$$
|B| \leq 2 C(\epsilon) \delta t|| \nabla w_{m}^{\epsilon}\left\|_{L^{2}(Q)}\right\| 1_{Q} \|_{L^{2}(Q)} \leq C(\epsilon) \delta t .
$$

The last integral of (5.5) is treated similarly:

$$
\begin{aligned}
|D| & \leq\left|\int_{0}^{T-\delta t} \int_{t}^{t+\delta t} \int_{\Omega} \nabla w_{m}^{\epsilon}(s, .) \cdot \nabla\left(w_{m}^{\epsilon}(t+\delta t, .)-w_{m}^{\epsilon}(t, .)\right)\right| \\
& \leq 2 \int_{\Omega} \int_{0}^{T-\delta t}\left|\nabla w_{m}^{\epsilon}(\tau, .) \cdot\right|\left(\int_{t}^{t+\delta t}\left|\nabla w_{m}^{\epsilon}(s, .)\right| d s\right) d \tau d x \\
& \leq 2\left\|\nabla w_{m}^{\epsilon}\right\|_{L^{2}(Q)}\left(\int_{\Omega} \int_{0}^{T-\delta t}\left(\int_{t}^{t+\delta t}\left|\nabla w_{m}^{\epsilon}(s, .)\right| d s\right)^{2} d \tau d x\right)^{\frac{1}{2}} .
\end{aligned}
$$

Using Jensen's Inequality,

$$
\left(\int_{t}^{t+\delta t}\left|\nabla\left(w_{m}^{\epsilon}(s, .)\right)\right| d s\right)^{2} \leq \int_{t}^{t+\delta t}\left|\nabla\left(w_{m}^{\epsilon}(s, .)\right)\right|^{2} d s,
$$

hence we obtain

$$
|D| \leq 2|| \nabla w_{m}^{\epsilon} \|_{L^{2}(Q)} \sqrt{\int_{\Omega} \int_{0}^{T} \int_{t}^{t+\delta t}\left|\nabla w_{m}^{\epsilon}(s, .)\right|^{2}} \leq C(\epsilon) \sqrt{\delta t}
$$


The sum of (5.6), (5.7) and (5.8) gives

$$
\int_{0}^{T-\delta t} \int_{\Omega}\left|\left(w_{m}^{\epsilon}(t+\delta t, .)-w_{m}^{\epsilon}(t, .)\right)\left(u_{m}^{\epsilon}(t+\delta t, .)-u_{m}^{\epsilon}(t, .)\right)\right| \leq C(\epsilon)(\delta t+\sqrt{\delta t}) .
$$

Now, using the fact $\phi_{\epsilon}^{-1}$ is Lipschitz, there exist another constant $C(\epsilon)$ such that

$$
\left\|u_{m}^{\epsilon}(t+\delta t, .)-u_{m}^{\epsilon}(t, .)\right\|_{L^{2}(0, T-\delta t ; \Omega)} \leq C(\epsilon)(\sqrt{\delta t}+\delta t) .
$$

By the characterization theorem for $H^{1}(\Omega)$ (see [9]), since $V_{m} \subset H^{1}(\Omega)$, and $\left\|\nabla w_{m}^{\epsilon}\right\|_{L^{2}(Q)} \leq C$, we have for all open subset $\omega \subset \subset \Omega$

$$
\left\|u_{m}^{\epsilon}(t, x+\delta x)-u_{m}^{\epsilon}(t, x)\right\|_{L^{2}(0, T ; \omega)} \leq C(\epsilon) \delta x .
$$

Finally, we obtain

$$
\left\|u_{m}^{\epsilon}(t+\delta t, x+\delta x)-u_{m}^{\epsilon}(t, x)\right\|_{L^{2}(0, T-\delta t ; \omega)} \leq C(\epsilon)(\sqrt{\delta t}+\delta t+\delta x) .
$$

Third step: Passage to the limit $(m \longrightarrow+\infty)$.

By the estimate $\left\|w_{m}^{\epsilon}\right\|_{L^{2}\left(0, T ; H^{1}(\Omega)\right)} \leq C(\epsilon)$, by (5.11) and Fréchet-Kolmogorov compactness criterion, $\left(u_{m}^{\epsilon}\right)_{m}$ is relatively compact in the space $L^{2}\left(0, T ; H^{1}(\Omega)\right)$ weakly and in $L_{\text {Loc }}^{1}((0, T) \times \Omega)$ strongly. Moreover, $\left\|u_{m}^{\epsilon}\right\|_{L^{2}(Q)} \leq C(\epsilon)$, then $u_{m}^{\epsilon}$ is relatively compact in $L^{1}(Q)$ strongly.

We can take $\xi(t) e_{i}(x)$ as test function in the weak formulation where $\xi \in \mathcal{D}[0, T)$, for $m \geq 0$, we have

$$
\int_{0}^{T} \int_{\Omega} u_{m}^{\epsilon} \xi^{\prime} e_{i} d x d t+\int_{0}^{T} \int_{\Omega}\left(f\left(u_{m}^{\epsilon}\right)-\nabla w_{m}^{\epsilon}\right) \cdot \nabla e_{i} \xi d x d t+\int_{\Omega} u_{0 m}^{\epsilon} \xi(0) e_{i} d x=0 .
$$

We can extract a subsequence $w_{m}^{\epsilon}$ such that $\nabla w_{m}^{\epsilon} \rightarrow \nabla w^{\epsilon}$ in $L^{2}(Q)$ and $u_{m}^{\epsilon} \rightarrow u^{\epsilon}$ in $L^{1}(Q)$ and a.e.. The Lebesgue theorem, continuity and boundedness of $f$ permit at last to pass to the limit. Finally we conclude that $(2.2)$ holds, this means that $u^{\epsilon}$ is a weak solution of $\left(P_{\epsilon}\right)$ by the density of the linear span of $\mathcal{D}(0, T) \times \mathcal{D}(\Omega)$ in $\mathcal{D}([0, T) \times \Omega)$.

At this point, we can also drop the Lipschitz continuity assumption on $\phi$. Indeed, approximating $\phi$ with a sequence of Lipschitz continuous functions $\phi_{\alpha}$, one can have uniform estimates in $\alpha$ (5.11). Then one can pass to the limit as $\alpha$ goes to zero in the equation corresponding to $\phi=\phi_{\alpha}$, with the same argument as above.

Its remains to prove that $u^{\epsilon}$ is an entropy solution.

Fourth step: Now, we prove that weak solution of $\left(P_{\epsilon}\right)$ is also an entropy sub-solution and entropy super-solution.

Since $u^{\epsilon}$ is a weak solution of $\left(P_{\epsilon}\right)$, then $u^{\epsilon}-k$ is a weak solution of the following problem

$$
\left(P_{k, \epsilon}\right)\left\{\begin{aligned}
\left(u^{\epsilon}-k\right)_{t}+\operatorname{div}\left[f\left(u^{\epsilon}\right)-\nabla \phi_{\epsilon}\left(u^{\epsilon}\right)-f(k)\right] & =0 & & \text { in } Q, \\
u^{\epsilon}(0, x)-k & =u_{0}^{\epsilon}(x)-k & & \text { in } \Omega, \\
\left(f\left(u^{\epsilon}\right)-\nabla \phi_{\epsilon}\left(u^{\epsilon}\right)-f(k)\right) \cdot \eta & =-f(k) . \eta & & \text { on } \Sigma .
\end{aligned}\right.
$$

Take $\operatorname{sign}_{\sigma}^{+}\left(u^{\epsilon}-k\right) \xi$ in the weak formulation of this problem with $\xi \in \mathcal{C}^{\infty}\left(\left[0, T\left[\times \mathbb{R}^{N}\right), \xi \geq 0\right.\right.$. We get (see [1] and [14] for the use of $H^{1}(\Omega)^{*}-H^{1}(\Omega)$ duality)

$$
\begin{aligned}
& \int_{0}^{T}\left\langle\left(u^{\epsilon}-k\right)_{t}, \operatorname{sign}_{\sigma}^{+}\left(u^{\epsilon}-k\right) \xi\right\rangle_{H^{1}(\Omega)^{*}, H^{1}(\Omega)}-\int_{0}^{T} \int_{\partial \Omega} \operatorname{sign}_{\sigma}^{+}\left(u^{\epsilon}-k\right) f(k) \cdot \eta \xi \\
& -\int_{0}^{T} \int_{\Omega}\left[f\left(u^{\epsilon}\right)-\nabla \phi_{\epsilon}\left(u^{\epsilon}\right)-f(k)\right] \xi \nabla \operatorname{sign}_{\sigma}^{+}\left(u^{\epsilon}-k\right) \\
& -\int_{0}^{T} \int_{\Omega} \operatorname{sign}_{\sigma}^{+}\left(u^{\epsilon}-k\right)\left[f\left(u^{\epsilon}\right)-\nabla \phi_{\epsilon}\left(u^{\epsilon}\right)-f(k)\right] \cdot \nabla \xi=0 .
\end{aligned}
$$


The first integral of 5.12 gives (see [1] and [14] for the use of Chain rule)

$$
\begin{aligned}
\int_{0}^{T}\left\langle\left(u^{\epsilon}-k\right)_{t}, \operatorname{sign}_{\sigma}^{+}\left(u^{\epsilon}-k\right) \xi\right\rangle & =-\int_{0}^{T} \int_{\Omega}\left\{\int_{k}^{u^{\epsilon}} \operatorname{sign}_{\sigma}^{+}(r-k) d r\right\} \xi_{t} d x d t \\
& -\int_{\Omega}\left\{\int_{k}^{u_{0}^{\epsilon}} \operatorname{sign}_{\sigma}^{+}(r-k) d r\right\} \xi(0, x) d x
\end{aligned}
$$

Passing to the limit as $\sigma$ goes to 0 , we obtain:

$$
\begin{aligned}
& \int_{0}^{T} \int_{\Omega}\left\{\int_{k}^{u^{\epsilon}} \operatorname{sign}_{\sigma}^{+}(r-k) d r\right\} \xi_{t} d x d t \longrightarrow \int_{0}^{T} \int_{\Omega}\left(u^{\epsilon}-k\right)^{+} \xi_{t} d x d t, \\
& \int_{\Omega}\left\{\int_{k}^{u_{0}^{\epsilon}} \operatorname{sign}_{\sigma}^{+}(r-k) d r\right\} \xi(0, x) d x \longrightarrow \int_{\Omega}\left(u_{0}^{\epsilon}-k\right)^{+} \xi(0, x) d x .
\end{aligned}
$$

The limit of the second integral of (5.12) can be upper bounded as follows:

$$
\lim _{\sigma \rightarrow 0} \int_{0}^{T} \int_{\partial \Omega} \operatorname{sign}_{\sigma}^{+}\left(u^{\epsilon}-k\right) f(k) \cdot \eta \xi \leq \int_{0}^{T} \int_{\partial \Omega}(f(k) \cdot \eta(x))^{+} \xi .
$$

The third integral of (5.12) can be written as

$$
-\iint_{\Omega} \xi \nabla \phi_{\epsilon}\left(u^{\epsilon}\right) \cdot \nabla \operatorname{sign}_{\sigma}^{+}\left(u^{\epsilon}-k\right)+\iint_{\Omega} \xi\left(f\left(u^{\epsilon}\right)-f(k)\right) \cdot \nabla \operatorname{sign}_{\sigma}^{+}\left(u^{\epsilon}-k\right) .
$$

Here, the first integral is non-positive. Moreover, the second one tends to zero as $\sigma \rightarrow 0$. In fact, we set $F_{\sigma}(r)=\int_{k-\sigma}^{r}(f(s)-f(k)) \operatorname{sign}_{\sigma}^{+^{\prime}}(s-k) d s$. We have $\left|F_{\sigma}(r)\right| \leq 2 \sup _{|k-s|<\sigma}|f(s)-f(k)|$. Using the Green-Gauss formula, we find

$$
\begin{aligned}
& \left|\int_{\Omega} \operatorname{sign}_{\sigma}^{+^{\prime}}\left(u^{\epsilon}-k\right) \nabla u^{\epsilon} \cdot\left(f\left(u^{\epsilon}\right)-f(k)\right) \xi\right|=\left|\int_{\Omega} \operatorname{div}\left(F_{\sigma}\left(u^{\epsilon}\right)\right) \xi\right| \\
& \leq 2 \sup _{|k-s|<\sigma}|f(s)-f(k)|\left(\int_{\Omega}|\nabla \xi|+\int_{\partial \Omega}|\xi|\right) \rightarrow 0 \text { as } \sigma \rightarrow 0 .
\end{aligned}
$$

Finally, we obtain

$$
\begin{aligned}
& \int_{0}^{T} \int_{\Omega}\left\{\left(u^{\epsilon}-k\right)^{+} \xi_{t}+\operatorname{sign}^{+}\left(u^{\epsilon}-k\right)\left[f\left(u^{\epsilon}\right)-\nabla \phi_{\epsilon}\left(u^{\epsilon}\right)-f(k)\right] . \nabla \xi\right\} d x d t \\
& +\int_{\Omega}\left(u_{0}^{\epsilon}-k\right)^{+} \xi(0, x) d x+\int_{0}^{T} \int_{\partial \Omega}(f(k) . \eta(x))^{+} \xi d \mathcal{H}^{n-1} d t \geq 0 .
\end{aligned}
$$

Therefore $u^{\epsilon}$ is entropy sub-solution of $\left(P_{\epsilon}\right)$. In the same way, we prove that $u^{\epsilon}$ is entropy supersolution of $\left(P_{\epsilon}\right)$.

Now we prove that $u^{\epsilon}$ is bounded. To this aim take $\xi=\xi(t)$, (i.e., $\nabla \xi=0$ ), take $k=0$ in (2.3), and use (1.1) and the fact that $u_{0}^{\epsilon} \in\left[0, u_{\max }\right]$. We get

$$
\int_{\Omega}\left(u^{\epsilon}\right)^{-}(0, .) \xi(0, .)+\int_{0}^{T} \int_{\Omega}\left(u^{\epsilon}\right)^{-}(t, x) \xi_{t}=\int_{0}^{T}\left(\int_{\Omega}\left(\left(u^{\epsilon}\right)^{-}(t, x)-\left(u_{0}^{\epsilon}\right)^{-}(x)\right)\right) \xi_{t} \geq 0 .
$$

Let us introduce the function

$$
G(t)=\left\{\begin{aligned}
\int_{\Omega}\left(\left(u^{\epsilon}\right)^{-}(t, x)-\left(u_{0}^{\epsilon}\right)^{-}(x)\right) d x & \text { for } t \in(0, T), \\
0 & \text { for } t \in(-T, 0) .
\end{aligned}\right.
$$

We have $\frac{d G}{d t} \leq 0$ in $\mathcal{D}^{\prime}(-T, T)$ and therefore, since $\mathrm{G}(\mathrm{t})$ vanishes for $t<0$, we deduce that

$$
\int_{\Omega}\left(u^{\epsilon}\right)^{-}(t, x) d x \leq \int_{\Omega}\left(u_{0}^{\epsilon}\right)^{-}(x) d x=0, \text { i.e., } u^{\epsilon}(t, x) \geq 0 .
$$


In the same way, we prouve that $u^{\epsilon}(t, x) \leq u_{\max }$.

Now, we go back to the technique used to get (5.3), recall that we can rewrite $\theta_{\epsilon}\left(w^{\epsilon}\right)$ as $\psi\left(u^{\epsilon}\right)$. We find

$$
\int_{\Omega} \psi_{\epsilon}\left(u^{\epsilon}\right)(t)+\left\|\nabla w^{\epsilon}\right\|_{L^{2}(0, t ; \Omega)}^{2} \leq C+\int_{\Omega} \psi_{\epsilon}\left(u^{\epsilon}\right)(0)
$$

The last term is now bounded uniformly in $\epsilon$, due to the $L^{\infty}$ bound on $u^{\epsilon}$. Therefore,

$$
\left\|w^{\epsilon}\right\|_{L^{2}\left(0, T ; H^{1}(\Omega)\right)} \leq C,
$$

with $C$ that is now $\epsilon$-independent.

Finally, if we take $u^{\epsilon}$ as test fonction in (3.1), we find

$$
\frac{1}{2}\left\|u^{\epsilon}(t)\right\|_{L^{2}(\Omega)}^{2}+\left\|\sqrt{\epsilon} \nabla u^{\epsilon}\right\|_{L^{2}(Q)}^{2} \leq C+\frac{1}{2}\left\|u_{0}^{\epsilon}\right\|_{L^{2}(\Omega)}^{2} .
$$

Then

$$
\left\|\sqrt{\epsilon} \nabla u^{\epsilon}\right\|_{L^{2}(Q)}^{2} \leq C .
$$

This concludes the proof of the Theorem.

\section{Acknowledgment}

The work on this paper has been supported by the French ANR project CoToCoLa. The authors thank G. Vallet for stimulating discussions.

\section{References}

[1] H.W. Alt, S. Luckhaus, Quasilinear elliptic-parabolic differential equations. Math. Z. 183 (3) (1983) 311-341.

[2] B. Andreianov, F. Bouhsiss, Uniqueness for an elliptic-parabolic problem with Neumann boundary condition. J. Evol. Equ. 4 (2004) 273-295.

[3] B. Andreianov, N. Igbida, On uniqueness techniques for degenerate convection-diffusion problems. Int. J. Dyn. Syst. Diff. Equ. ,4(112 (2012) 3-34.

[4] B. Andreianov, K. Shibi, Scalar conservation laws with nonlinear boundary conditions C. R. Acad. Paris. 345 (8) (2007) 431-434.

[5] C. Bardos, A.Y. Le Roux, J.C. Nedelec, First order quasilinear equations with boundary conditions. Comm. PDE. 4 (1979) 1017-1034.

[6] Ph. Bénilan, Crandall, M. G. and Pazy, A., Nonlinear evolution equations in Banach spaces. Preprint book.

[7] Ph., Bénilan, H., Toure, Sur l'équation générale $u_{t}=a\left(., u, \phi(., u)_{x}\right)_{x}+v$ dans $L^{1}$. I. Etude du probleme stationnaire, In Evolution equations (Baton Rouge, LA, 1992). pp. 35.62. Dekker, New York, 1995.

[8] Ph. Bénilan, P. Wittbold, On mild and weak solutions of elliptic-parabolic problems. Adv. Differential Equations. 1 (6) (1996) 1053-1073.

[9] H. Brezis, Functional Analysis, Sobolev Space and Partial Differential Equation. Spring-Verlag New York Inc, (2010).

[10] R. Bürger, H. Frid, K. H. Karlsen, On the well-posedness of entropy solution to conservation laws with a zero-flux boundary condition. J. Math. Anal. Appl. 326 (2007), 108-120.

[11] R. Bürger, H. Frid, K. H. Karlsen, On a free boundary problem for a strongly degenerate quasilinear parabolic equation with an application to a model of presssure filtration. SIAM J. Math. Anal. 34 (2003) 611-635.

[12] C. Cancés, T. Gallouët, On the time continuity of entropy solutions. J. Evol Equ 11 (2011) 43-45.

[13] J. Carrillo, Entropy solutions for nonlinear degenerate problems. Arch. Ration. Mech. Anal. 147 (4) (1999) 269-361.

[14] G. Gagneux , M. Madaune-Tort, Analyse mathématique de modeles nonlinéaires de l'ingégnerie pétroliere; Mathématique et Applications, 22. Springer-Verlag, Berlin, (1996).

[15] S.N. Kruzkhov, First order quasi-linear equations in several independent variables. Math. USSR Sb. 10 (2) (1970) 217-243.

[16] J.L. Lions, Quelques méthodes de résolution des problémes aux limites non linéaires. Dunod, (1969). 
[17] C. Mascia, A. Porretta, A. Terracina, Nonhomogeneous Dirichlet problems for degenerate parabolichyperbolic equation. Arch. Ration. Mech. Anal. 163 (2) (2002) 87-124.

[18] M. Marcus, V.J. Mizel, Absolute continuity on Tracks and Mappings of Sobolev Spaces. Arch. Ration Mech. Anal. 45, (4) (1972), 294-320.

[19] A. Michel, J. Vovelle, Entropy formulation for parabolic degenerate equations with general Dirichlet boundary conditions and application to the convergence of FV methods. SIAM J. Numer. Anal. 41 (2003) 22622293.

[20] E.Yu. Panov, On the strong pre-compactness property for entropy solutions of a degenerate elliptic equation with discontinuous flux. J. Differential Equations. 247 (2009) 28212870.

[21] E. Rouvre and G. Gagneux, Formulation forte entropique de lois scalaires hyperboliques-paraboliques dgnres. An. Fac. Sci. Toulouse. X (1) (2001) 163-183.

[22] G. Vallet, Dirichlet problem for a degenerated hyperbolic-parabolic equation. Advance in Math. Sci. Appl. 15 (2) (2005)

[23] A. Vasseur, Strong traces for solutions of multidimensional scalar conservation laws. Arch. Ration. Mech. Anal. 160 (2001) 181-193.

Boris Andreianov

Laboratoire de Mathématiques

CNRS : UMR 6623 - Université de Franche-Comte

16 , route de Gray

25030 Besancon France

e-mail: boris .andreianov@univ-fcomte.fr

Mohamed Karimou Gazibo

Laboratoire de Mathématiques

CNRS : UMR 6623 - Université de Franche-Comte

16 , route de Gray

25030 Besancon France

e-mail: mgazibok@univ-fcomte.fr 